DOI 10.4171/JEMS/225

Olivier Druet · Paul Laurain

\title{
Stability of the Pohožaev obstruction in dimension 3
}

Received June 26, 2008 and in revised form October 2, 2008

Abstract. We investigate problems connected to the stability of the well-known Pohožaev obstruction. We generalize results which were obtained in the minimizing setting by Brezis and Nirenberg [2] and more recently in the radial situation by Brezis and Willem [3].

Let $\Omega$ be a smooth bounded domain in $\mathbb{R}^{n}, n \geq 3$. Let $h \in C^{1}\left(\mathbb{R}^{n}\right)$ and consider the equation

$$
\left\{\begin{array}{l}
\Delta u+h u=|u|^{4 /(n-2)} u \quad \text { in } \Omega \\
u=0 \text { on } \partial \Omega
\end{array}\right.
$$

where $\Delta u=-\sum_{i=1}^{n} \partial^{2} u / \partial x_{i}^{2}$. It is well-known that if $\Omega$ is star-shaped with respect to the origin and if $h$ satisfies

$$
h(x)+\frac{1}{2}\langle x, \nabla h(x)\rangle \geq 0,
$$

then there are no non-trivial solutions of (0.1). This is a consequence of Pohožaev's identity (see [11] and equation (4.6) of appendix 4.3) and is referred to as the Pohožaev obstruction.

The above equation has been quite intensively studied in the past thirty years. Many existence results have been obtained if $\Omega$ is not assumed to be star-shaped or if $h$ does not satisfy (0.2). It is almost impossible to give an exhaustive list of references on this equation.

In this paper, we investigate the question of non-existence of positive solutions of equation 0.1 and more precisely the stability properties of the Pohožaev obstruction.

Definition 0.1. Let $\Omega$ be a star-shaped domain of $\mathbb{R}^{n}$ and let $\left(X,\|\cdot\|_{X}\right)$ be some Banach space of functions on $\Omega$ (typically $X=\mathcal{C}^{k, \eta}(\Omega), X=L^{\infty}(\Omega)$ or $X=L^{p}(\Omega)$ ). Let $h_{0} \in X \cap C^{1}(\Omega)$ be a function which satisfies $(0.2)$. We say that the Pohožaev obstruction is $X$-stable at $\left(h_{0}, \Omega\right)$ if the following property holds: there exists $\delta\left(h_{0}, \Omega, X\right)>0$ such that for any function $h \in X$ with

$$
\left\|h-h_{0}\right\|_{X} \leq \delta\left(h_{0}, \Omega, X\right),
$$

the only non-negative $C^{2}$-solution of $(0.1)$ is $u \equiv 0$.

O. Druet, P. Laurain: Département de mathématiques, UMPA, École normale supérieure de Lyon, 46 allée d'Italie, 69364 Lyon Cedex 07, France; e-mail: Olivier.Druet@umpa.ens-lyon.fr; Paul.Laurain@umpa.ens-lyon.fr 
We say that the Pohožaev obstruction is $X$-stable if it is $X$-stable at $\left(h_{0}, \Omega\right)$ for all $\Omega$ star-shaped with respect to the origin and all $h_{0} \in X \cap C^{1}(\Omega)$ satisfying 0.2 .

Note that the property 0.2 is not stable under perturbations of the function $h$ in any $C^{k}$-space. Since the work of Brezis and Nirenberg [2], we know that equation 0.1 behaves differently in dimension 3 and in dimensions $n \geq 4$. It is clear that, in dimensions $n \geq 4$, the Pohožaev obstruction is not $X$-stable for any reasonable $X$. Indeed, any perturbation of $h \equiv 0$ which is negative somewhere leads to a minimizing solution in dimensions $n \geq 4$ (see [2]) 1 Hence we investigate the stability of the Pohožaev obstruction for various spaces $X$ in dimension 3 . We give a complete answer to this problem in the following theorems.

Theorem 1. The Pohožaev obstruction is $C^{0, \eta}$-stable for any $\eta>0$ in dimension 3. In other words, given any $\eta>0$, any domain $\Omega$ in $\mathbb{R}^{3}$, star-shaped with respect to the origin, and any function $h_{0} \in C^{1}(\Omega)$ satisfying $(0.2)$, there exists $\delta\left(\eta, \Omega, h_{0}\right)>0$ such that if $h \in C^{0, \eta}(\Omega)$ satisfies

$$
\left\|h-h_{0}\right\|_{C^{0, \eta}(\Omega)} \leq \delta(\eta, \Omega, h),
$$

the only non-negative solution of $[0.1)$ is $u \equiv 0$.

Note that a consequence of our theorem is the following: if $\Omega$ is a star-shaped domain in $\mathbb{R}^{3}$, there exists a constant $\hat{\lambda}(\Omega)>0$ such that equation 0.1 does not possess any non-trivial positive solutions with $h \equiv \lambda$ for $\lambda>-\hat{\lambda}(\Omega)$. This is in sharp contrast with the situation for non-star-shaped domains (see [1] for instance).

In the seminal paper [2], it was proved that there are no minimizing solutions of equation 0.1 in dimension 3 if $h \geq-\lambda^{\star}(\Omega)$ for some $\lambda^{\star}(\Omega)>0$. Since $h \geq 0$ if $h$ satisfies 0.2 , a consequence of this result is a version of the above stability in $C^{0}$ when one considers only minimizing solutions. A necessary and sufficient condition on the function $h$ and the domain $\Omega$ for the existence of a minimizing solution of $[0.1)$ in dimension 3 was found in [6].

In [3], the authors studied this question in the case of the unit ball with radial functions. If we let

$$
L_{r}^{p}(B)=\left\{u \in L^{p}(B): u \text { radial }\right\},
$$

then it was proved in [3] that the Pohožaev obstruction is $L_{r}^{\infty}$-stable ${ }^{2}$ on the unit ball of $\mathbb{R}^{3}$ for all $h \in L_{r}^{\infty}(B) \cap C^{1}(B)$. In [3], the question of extending the result to the non-radial case was explicitly asked. Our result provides an answer to this question. However, the situation is more delicate than expected in the non-radial case since, while the Pohožaev obstruction is $C^{0, \eta}$-stable for all $\eta>0$, it is never $L^{\infty}$-stable.

Theorem 2. The Pohožaev obstruction is never $L^{\infty}$-stable. In other words, given any $\varepsilon>0$, any domain $\Omega$ in $\mathbb{R}^{3}$, star-shaped with respect to the origin, and any function $h_{0} \in C^{1}(\Omega)$ satisfying 0.2 , we can find some function $h_{\varepsilon} \in L^{\infty}(\Omega)$ such that

$$
\left\|h_{\varepsilon}-h\right\|_{\infty} \leq \varepsilon
$$

\footnotetext{
1 Note that this remark concerns only $X$-stability in general. The question of $X$-stability at some given positive function $h$ in dimensions $n \geq 4$ is not investigated in this paper.

2 One should restrict oneself to radial solutions of the equation in the definition of stability.
} 
and some positive functions $u_{\varepsilon} \in C^{2}(\Omega)$ satisfying the equation

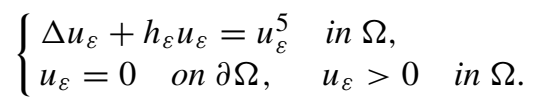

Thus the $L_{r}^{\infty}$-stability result obtained by Brezis-Willem is really specific to the radial case. In fact, it is not due to the symmetry of the solutions but to one of its by-products in dimension 3, precisely that sequences of solutions of equation 0.1 which are radial are either compact or develop only one concentration point. In fact, with the PDE techniques (as compared to the ODE techniques used in [3]) we use below, we can revisit the question of the stability of the Pohožaev obstruction in dimension 3 in the radial case. We improve the result of [3] by proving that the Pohožaev obstruction is $L_{r}^{p}$-stable on the unit ball for all $p>3$ but is never $L_{r}^{3}$-stable. For precise statements, we refer the reader to the end of Section 2 and the beginning of Section 3

All these results give a complete picture of the stability of the Pohožaev obstruction in dimension 3 when the attention is restricted to non-negative solutions. The question remains widely open if one allows solutions to change sign, and is certainly more subtle due to the variety of changing-sign solutions of $\Delta u=u^{5}$ in $\mathbb{R}^{3}$.

The paper is organized as follows. Section 2 is devoted to the proofs of Theorem 1 and of the corresponding result in the radial situation. The proof makes use of standard blowup analysis in dimension 3 (see Section 1) and of an extension of Pohožaev's identity to Green's functions (see Appendix 4.4). Section 3 is devoted to the proofs of Theorem 2 and of the corresponding result in the radial situation. Here we have to construct examples of functions $h$ arbitrarily close in $X$ to some given function for which there is a positive solution of equation 0.1 . This appears to be quite subtle because we need to be sharp. For instance, in order to prove Theorem 2 , our functions $h$ must be close to the given function in $L^{\infty}(\Omega)$ but not in $C^{0, \eta}(\Omega)$ for any $\eta>0$.

\section{Pointwise analysis around a concentration point}

In this section we consider a sequence $\left(h_{\varepsilon}\right)$ in $C^{0, \eta}\left(\mathbb{R}^{3}\right)$ for some $\eta>0$ and a sequence $\left(u_{\varepsilon}\right)$ of $C^{2}$-solutions of

$$
\begin{cases}\Delta u_{\varepsilon}+h_{\varepsilon} u_{\varepsilon}=u_{\varepsilon}^{5} & \text { in } \Omega, \\ u_{\varepsilon}=0 & \text { on } \partial \Omega, \\ u_{\varepsilon}>0 & \text { in } \Omega,\end{cases}
$$

where $\Omega$ is some smooth domain in $\mathbb{R}^{3}$ and

$$
h_{\varepsilon} \rightarrow h \quad \text { in } L^{p}(\Omega) \text { as } \varepsilon \rightarrow 0
$$

for some $p>3$ where $h \in C^{1}\left(\mathbb{R}^{3}\right)$ satisfies $h \geq 0$ in $\Omega$. Note that, as soon as $h$ satisfies $[0.2]$, it is non-negative.

We also assume that we have a sequence $\left(x_{\varepsilon}\right)$ of points in $\Omega$ and a sequence $\left(\rho_{\varepsilon}\right)$ of positive real numbers with $0<3 \rho_{\varepsilon} \leq d\left(x_{\varepsilon}, \partial \Omega\right)$ such that

$$
\nabla u_{\varepsilon}\left(x_{\varepsilon}\right)=0
$$


and

$$
\rho_{\varepsilon}\left[\sup _{B\left(x_{\varepsilon}, \rho_{\varepsilon}\right)} u_{\varepsilon}(x)\right]^{2} \rightarrow+\infty \quad \text { as } \varepsilon \rightarrow 0 .
$$

We prove the following:

Proposition 1.1. If there exists $C_{0}>0$ such that

$$
\left|x_{\varepsilon}-x\right|^{1 / 2} u_{\varepsilon} \leq C_{0} \quad \text { in } B\left(x_{\varepsilon}, 3 \rho_{\varepsilon}\right),
$$

then there exists $C_{1}>0$ such that

$$
\begin{array}{ll}
u_{\varepsilon}\left(x_{\varepsilon}\right) u_{\varepsilon}(x) \leq C_{1}\left|x_{\varepsilon}-x\right|^{-1} & \text { in } B\left(x_{\varepsilon}, 2 \rho_{\varepsilon}\right) \backslash\left\{x_{\varepsilon}\right\}, \\
u_{\varepsilon}\left(x_{\varepsilon}\right)\left|\nabla u_{\varepsilon}(x)\right| \leq C_{1}\left|x_{\varepsilon}-x\right|^{-2} & \text { in } B\left(x_{\varepsilon}, 2 \rho_{\varepsilon}\right) \backslash\left\{x_{\varepsilon}\right\} .
\end{array}
$$

Moreover, if $\rho_{\varepsilon} \rightarrow 0$, then

$$
\rho_{\varepsilon} u_{\varepsilon}\left(x_{\varepsilon}\right) u_{\varepsilon}\left(x_{\varepsilon}+\rho_{\varepsilon} x\right) \rightarrow \frac{1}{|x|}+b \quad \text { in } C_{\mathrm{loc}}^{1}(B(0,2) \backslash\{0\}) \text { as } \varepsilon \rightarrow 0
$$

where $b$ is some harmonic function in $B(0,2)$ with $b(0)=0$. Finally, if the convergence in 1.2 holds in $C^{0, \eta}$, then also $\nabla b(0)=0$.

The rest of this section is dedicated to the proof of this proposition. We follow the lines of [7, Section 2] (see also [8]). However, one must note that, compared to [8] and other works on this kind of blow-up analysis, some new difficulties arise since the linear term $\left(h_{\varepsilon}\right)$ is only uniformly bounded in some $L^{p}(\Omega)$.

We divide the proof of the proposition into several claims. The first one gives the asymptotic behaviour of $u_{\varepsilon}$ around $x_{\varepsilon}$ at an appropriate small scale.

Claim 1.1. After passing to a subsequence, we have

$$
\mu_{\varepsilon}^{1 / 2} u_{\varepsilon}\left(x_{\varepsilon}+\mu_{\varepsilon} x\right) \rightarrow \frac{1}{\left(1+|x|^{2} / 3\right)^{1 / 2}} \quad \text { in } C_{\mathrm{loc}}^{1}\left(\mathbb{R}^{3}\right) \text { as } \varepsilon \rightarrow 0
$$

where $\mu_{\varepsilon}=u_{\varepsilon}\left(x_{\varepsilon}\right)^{-2}$.

Proof. Let $\tilde{x}_{\varepsilon} \in \overline{B\left(x_{\varepsilon}, \rho_{\varepsilon}\right)}$ and $\tilde{\mu}_{\varepsilon}>0$ be such that

$$
u_{\varepsilon}\left(\tilde{x}_{\varepsilon}\right)=\sup _{B\left(x_{\varepsilon}, \rho_{\varepsilon}\right)} u_{\varepsilon}=\tilde{\mu}_{\varepsilon}^{-1 / 2} .
$$

Thanks to (1.4), we have

$$
\tilde{\mu}_{\varepsilon} \rightarrow 0 \quad \text { and } \quad \rho_{\varepsilon} / \tilde{\mu}_{\varepsilon} \rightarrow+\infty \quad \text { as } \varepsilon \rightarrow 0 .
$$

By (1.5), we also have

$$
\left|x_{\varepsilon}-\tilde{x}_{\varepsilon}\right|=O\left(\tilde{\mu}_{\varepsilon}\right) .
$$

For $x \in \Omega_{\varepsilon}=\left\{x \in \mathbb{R}^{3}: \tilde{x}_{\varepsilon}+\tilde{\mu}_{\varepsilon} x \in \Omega\right\}$, we set

$$
\tilde{u}_{\varepsilon}(x)=\tilde{\mu}_{\varepsilon}^{1 / 2} u_{\varepsilon}\left(\tilde{x}_{\varepsilon}+\tilde{\mu}_{\varepsilon} x\right),
$$


which satisfies

$$
\begin{aligned}
& \Delta \tilde{u}_{\varepsilon}+\tilde{\mu}_{\varepsilon}^{2} \tilde{h}_{\varepsilon} \tilde{u}_{\varepsilon}=\tilde{u}_{\varepsilon}^{5} \quad \text { in } \Omega_{\varepsilon}, \\
& \tilde{u}_{\varepsilon}(0)=\sup _{B\left(\left(x_{\varepsilon}-\tilde{x}_{\varepsilon}\right) / \tilde{\mu}_{\varepsilon}, \rho_{\varepsilon} / \tilde{\mu}_{\varepsilon}\right)} \tilde{u}_{\varepsilon}=1,
\end{aligned}
$$

where $\tilde{h}_{\varepsilon}=h\left(\tilde{x}_{\varepsilon}+\tilde{\mu}_{\varepsilon} x\right)$. Thanks to 1.4, 1.7 and 1.9 , we get

$$
B\left(\frac{x_{\varepsilon}-\tilde{x}_{\varepsilon}}{\tilde{\mu}_{\varepsilon}}, \frac{\rho_{\varepsilon}}{\tilde{\mu}_{\varepsilon}}\right) \rightarrow \mathbb{R}^{3} \quad \text { as } \varepsilon \rightarrow 0 .
$$

Now, from 1.10 , 1.11 , and by standard elliptic theory, we find that, after passing to a subsequence, $\tilde{u}_{\varepsilon} \rightarrow U$ in $C_{\text {loc }}^{1}\left(\mathbb{R}^{3}\right)$ as $\varepsilon \rightarrow 0$ where $U$ satisfies

$$
\Delta U=U^{5} \quad \text { in } \mathbb{R}^{3} \quad \text { and } \quad 0 \leq U \leq 1=U(0) .
$$

From the work of Caffarelli, Gidas and Spruck [4], we know that

$$
U(x)=\left(1+|x|^{2} / 3\right)^{-1 / 2} .
$$

Moreover, thanks to 1.9 , after passing to a new subsequence, $\left(x_{\varepsilon}-\tilde{x}_{\varepsilon}\right) / \tilde{\mu}_{\varepsilon} \rightarrow x_{0}$ as $\varepsilon \rightarrow 0$ for some $x_{0} \in \mathbb{R}^{3}$. Hence, since $x_{\varepsilon}$ is a critical point of $u_{\varepsilon}, x_{0}$ must be a critical point of $U$, hence $x_{0}=0$. We deduce that $\mu_{\varepsilon} / \tilde{\mu}_{\varepsilon} \rightarrow 1$ where $\mu_{\varepsilon}$ is as in the statement of the claim, so the claim follows.

For $0 \leq r \leq 3 \rho_{\varepsilon}$, we set

$$
\psi_{\varepsilon}(r)=\frac{r^{1 / 2}}{\omega_{2} r^{2}} \int_{\partial B\left(x_{\varepsilon}, r\right)} u_{\varepsilon} d \sigma
$$

where $d \sigma$ denotes the Lebesgue measure on the sphere $\partial B\left(x_{\varepsilon}, r\right)$ and $\omega_{2}=4 \pi$ is the volume of the unit 2 -sphere. We easily check, thanks to Claim 1.1, that

$\psi_{\varepsilon}\left(\mu_{\varepsilon} r\right)=\left(\frac{r}{1+r^{2} / 3}\right)^{1 / 2}+o(1), \quad \psi_{\varepsilon}^{\prime}\left(\mu_{\varepsilon} r\right)=\frac{1}{2}\left(\frac{r}{1+r^{2} / 3}\right)^{3 / 2}\left(\frac{1}{r^{2}}-\frac{1}{3}\right)+o(1)$.

We define

$$
r_{\varepsilon}=\max \left\{r \in\left[2 \sqrt{3} \mu_{\varepsilon}, \rho_{\varepsilon}\right]: \psi_{\varepsilon}^{\prime}(s) \leq 0 \text { for } s \in\left[2 \sqrt{3} \mu_{\varepsilon}, r\right]\right\} .
$$

Thanks to 1.12, the set on which the maximum is attained is not empty for $\varepsilon$ small enough, and moreover

$$
r_{\varepsilon} / \mu_{\varepsilon} \rightarrow+\infty \quad \text { as } \varepsilon \rightarrow 0
$$

We now prove the following:

Claim 1.2. There exists $C>0$, independent of $\varepsilon$, such that

$$
\begin{array}{ll}
u_{\varepsilon}(x) \leq C \mu_{\varepsilon}^{1 / 2}\left|x_{\varepsilon}-x\right|^{-1} & \text { in } B\left(x_{\varepsilon}, 2 r_{\varepsilon}\right) \backslash\left\{x_{\varepsilon}\right\}, \\
\left|\nabla u_{\varepsilon}(x)\right| \leq C \mu_{\varepsilon}^{1 / 2}\left|x_{\varepsilon}-x\right|^{-2} & \text { in } B\left(x_{\varepsilon}, 2 r_{\varepsilon}\right) \backslash\left\{x_{\varepsilon}\right\} .
\end{array}
$$


Proof. We follow the proof of Lemmas 1.5 and 1.6 of [8]. However, there is an extra difficulty due to the fact that we do not assume any pointwise convergence of $h_{\varepsilon}$ to $h$. We first prove that for any given $0<v<1 / 2$, there exists $C_{v}>0$ such that

$$
u_{\varepsilon}(x) \leq C_{\nu}\left(\mu_{\varepsilon}^{\frac{1}{2}(1-2 v)}\left|x-x_{\varepsilon}\right|^{-(1-v)}+\alpha_{\varepsilon}\left(\frac{r_{\varepsilon}}{\left|x-x_{\varepsilon}\right|}\right)^{v}\right)
$$

for all $x \in B\left(x_{\varepsilon}, 2 r_{\varepsilon}\right)$ and $\varepsilon$ small enough, where

$$
\alpha_{\varepsilon}=\sup _{\partial B\left(x_{\varepsilon}, r_{\varepsilon}\right)} u_{\varepsilon}
$$

First of all, we can use (1.5) and apply the Harnack inequality (see for instance Theorem 4.17 of [10]) to get the existence of some $C>0$ such that

$$
\frac{1}{C} \max _{\partial B\left(x_{\varepsilon}, r\right)}\left(u_{\varepsilon}+r\left|\nabla u_{\varepsilon}\right|\right) \leq \frac{1}{\omega_{2} r^{2}} \int_{\partial B\left(x_{\varepsilon}, r\right)} u_{\varepsilon} d \sigma \leq C \min _{\partial B\left(x_{\varepsilon}, r\right)} u_{\varepsilon}
$$

for all $0<r<\frac{5}{2} \rho_{\varepsilon}$ and all $\varepsilon>0$. The details of the proof can be found in [8, Lemma 1.3]. Hence, thanks to (1.12) and (1.13), we have

$$
\left|x-x_{\varepsilon}\right|^{1 / 2} u_{\varepsilon}(x) \leq C \psi_{\varepsilon}(r) \leq C \psi_{\varepsilon}\left(R \mu_{\varepsilon}\right)=C\left(\frac{R}{1+R^{2} / 3}\right)^{1 / 2}+o(1)
$$

for all $R \geq 2 \sqrt{3}$, all $r \in\left[R \mu_{\varepsilon}, r_{\varepsilon}\right]$, all $\varepsilon$ small enough and all $x \in \partial B\left(x_{\varepsilon}, r\right)$. Thus

$$
\sup _{B\left(x_{\varepsilon}, r_{\varepsilon}\right) \backslash B\left(x_{\varepsilon}, R \mu_{\varepsilon}\right)}\left|x-x_{\varepsilon}\right|^{1 / 2} u_{\varepsilon}(x)=e(R)+o(1)
$$

where $e(R) \rightarrow 0$ as $R \rightarrow+\infty$. Let $0<\sigma \leq 1$ and $\mathcal{G}_{\varepsilon, \sigma}$ be the Green function of the operator $\Delta+h_{\varepsilon} / \sigma$ in $\Omega$ with Dirichlet boundary conditions. As $h$ is non-negative (this is an assumption in this section), we can use Lemma 4.2 of the Appendix to get the existence of some $C_{\sigma}>0$ such that

$$
|| x-y\left|\mathcal{G}_{\varepsilon, \sigma}(x, y)-\frac{1}{\omega_{2}}\right| \leq C_{\sigma}|x-y|
$$

and

$$
|| x-\left.y\right|^{2}\left|\nabla \mathcal{G}_{\varepsilon, \sigma}(x, y)\right|-\frac{1}{\omega_{2}}\left|\leq C_{\sigma}\right| x-y \mid
$$

for all $x \neq y \in \Omega$. We fix $0<v<1 / 2$ and set

$$
\Phi_{\varepsilon, v}=\mu_{\varepsilon}^{\frac{1}{2}(1-2 v)} \mathcal{G}_{\varepsilon, 1-v}\left(x_{\varepsilon}, x\right)^{1-v}+\alpha_{\varepsilon}\left(r_{\varepsilon} \mathcal{G}_{\varepsilon, v}\left(x_{\varepsilon}, x\right)\right)^{v} .
$$

Thanks to (1.18), (1.14) reduces to proving

$$
\sup _{B\left(x_{\varepsilon}, 2 r_{\varepsilon}\right)} \frac{u_{\varepsilon}}{\Phi_{\varepsilon, v}}=O(1) .
$$


We let $y_{\varepsilon} \in \overline{B\left(x_{\varepsilon}, 2 r_{\varepsilon}\right) \backslash\left\{x_{\varepsilon}\right\}}$ be such that

$$
\sup _{B\left(x_{\varepsilon}, 2 r_{\varepsilon}\right)} \frac{u_{\varepsilon}}{\Phi_{\varepsilon, v}}=\frac{u_{\varepsilon}\left(y_{\varepsilon}\right)}{\Phi_{\varepsilon, v}\left(y_{\varepsilon}\right)} .
$$

We are going to consider several possible behaviours of the sequence $\left(y_{\varepsilon}\right)$.

First of all, assume that

$$
\left|x_{\varepsilon}-y_{\varepsilon}\right| / \mu_{\varepsilon} \rightarrow R \quad \text { as } \varepsilon \rightarrow 0 .
$$

Thanks to Claim 1.1, in this case we have

$$
\mu_{\varepsilon}^{1 / 2} u_{\varepsilon}\left(y_{\varepsilon}\right) \rightarrow\left(1+R^{2}\right)^{-1 / 2} \quad \text { as } \varepsilon \rightarrow 0 .
$$

On the other hand, by 1.17 , we can write

$$
\begin{aligned}
\mu_{\varepsilon}^{1 / 2} \Phi_{\varepsilon, v}\left(y_{\varepsilon}\right) & =\left(\frac{\mu_{\varepsilon}}{\omega_{2}\left|x_{\varepsilon}-y_{\varepsilon}\right|}\right)^{1-\nu}+O\left(\alpha_{\varepsilon} \mu_{\varepsilon}^{1 / 2}\left(\frac{r_{\varepsilon}}{\left|x_{\varepsilon}-y_{\varepsilon}\right|}\right)^{\nu}\right)+o(1) \\
& =\left(R \omega_{2}\right)^{\nu-1}+O\left(\left(r_{\varepsilon}^{1 / 2} \alpha_{\varepsilon}\right) \mu_{\varepsilon}^{\frac{1}{2}(1-2 v)} r_{\varepsilon}^{\frac{1}{2}(2 v-1)}\right)+o(1)=\left(R \omega_{2}\right)^{\nu-1}+o(1)
\end{aligned}
$$

if $R>0$, and $\mu_{\varepsilon}^{1 / 2} \Phi_{\varepsilon, v}\left(y_{\varepsilon}\right) \rightarrow+\infty$ as $\varepsilon \rightarrow 0$ if $R=0$. In any case, $\left(u_{\varepsilon}\left(y_{\varepsilon}\right) / \Phi_{\varepsilon, v\left(y_{\varepsilon}\right)}\right)$ is bounded.

Assume now that there exists $\delta>0$ such that $y_{\varepsilon} \in B\left(y_{\varepsilon}, r_{\varepsilon}\right) \backslash B\left(y_{\varepsilon}, \delta r_{\varepsilon}\right)$. Thanks to Harnack's inequality (1.16), we get $u_{\varepsilon}\left(y_{\varepsilon}\right)=O\left(\alpha_{\varepsilon}\right)$, which, by (1.18), easily gives that $u_{\varepsilon}\left(y_{\varepsilon}\right) / \Phi_{\varepsilon, v\left(y_{\varepsilon}\right)}=O(1)$.

Hence, we are left with the following situation:

$$
\left|y_{\varepsilon}-x_{\varepsilon}\right| / r_{\varepsilon} \rightarrow 0 \quad \text { and } \quad\left|x_{\varepsilon}-y_{\varepsilon}\right| / \mu_{\varepsilon} \rightarrow+\infty \quad \text { as } \varepsilon \rightarrow 0 .
$$

By the definition of $y_{\varepsilon}$, we then have

$$
\frac{\Delta u_{\varepsilon}\left(y_{\varepsilon}\right)}{u_{\varepsilon}\left(y_{\varepsilon}\right)} \geq \frac{\Delta \Phi_{\varepsilon, v}\left(y_{\varepsilon}\right)}{\Phi_{\varepsilon, \eta}\left(y_{\varepsilon}\right)},
$$

which gives, thanks to the definition of $\Phi_{\varepsilon, v}$ and after multiplying by $\left|x_{\varepsilon}-y_{\varepsilon}\right|^{2}$,

$$
\begin{aligned}
\left|x_{\varepsilon}-y_{\varepsilon}\right|^{2} u_{\varepsilon}\left(y_{\varepsilon}\right)^{4} \geq & v(1-v) \frac{\left|x_{\varepsilon}-y_{\varepsilon}\right|^{2}}{\Phi_{\varepsilon, \eta}\left(y_{\varepsilon}\right)}\left(\alpha_{\varepsilon} r_{\varepsilon}^{\nu} \frac{\left|\nabla \mathcal{G}_{\varepsilon, v}\left(x_{\varepsilon}, y_{\varepsilon}\right)\right|^{2}}{\mathcal{G}_{\varepsilon, v}\left(x_{\varepsilon}, y_{\varepsilon}\right)^{2}} \mathcal{G}_{\varepsilon, v}\left(x_{\varepsilon}, y_{\varepsilon}\right)^{v}\right. \\
& \left.+\mu_{\varepsilon}^{\frac{1}{2}(1-2 \eta)} \frac{\left|\nabla \mathcal{G}_{\varepsilon, 1-v}\left(x_{\varepsilon}, y_{\varepsilon}\right)\right|^{2}}{\mathcal{G}_{\varepsilon, 1-v}\left(x_{\varepsilon}, y_{\varepsilon}\right)^{2}} \mathcal{G}_{\varepsilon, 1-v}\left(x_{\varepsilon}, y_{\varepsilon}\right)^{1-v}\right) .
\end{aligned}
$$

Here is the main difference with [8]. Thanks to our choice of $\Phi_{\varepsilon, v}$, the terms involving $h_{\varepsilon}$ disappear, which is necessary since we did not assume any pointwise convergence of $h_{\varepsilon}$. By (1.17), the left-hand side goes to 0 as $\varepsilon \rightarrow 0$. Then, thanks to (1.18)-1.20, we get

$$
o(1) \geq v(1-v)+o(1),
$$


which is a contradiction, and shows that this last case cannot occur. This ends the proof of (1.14).

We now claim that there exists $C>0$, independent of $\varepsilon$, such that

$$
u_{\varepsilon}(x) \leq C\left(\mu_{\varepsilon}^{1 / 2}\left|x-x_{\varepsilon}\right|^{-1}+\alpha_{\varepsilon}\right) \quad \text { in } B\left(x_{\varepsilon}, r_{\varepsilon}\right) .
$$

Thanks to Claim 1.1 and $(1.16)$, this holds for all sequences $y_{\varepsilon} \in B\left(x_{\varepsilon}, r_{\varepsilon}\right) \backslash\left\{x_{\varepsilon}\right\}$ such that $\left|y_{\varepsilon}-x_{\varepsilon}\right|=O\left(\mu_{\varepsilon}\right)$ or $\left|y_{\varepsilon}-x_{\varepsilon}\right| / r_{\varepsilon} \nrightarrow 0$. Thus we may assume from now on that

$$
\left|y_{\varepsilon}-x_{\varepsilon}\right| / \mu_{\varepsilon} \rightarrow+\infty \text { and }\left|y_{\varepsilon}-x_{\varepsilon}\right| / r_{\varepsilon} \rightarrow 0 \quad \text { as } \varepsilon \rightarrow 0 .
$$

Using the Green representation formula, we deduce from (1.18) and (1.19) that

$$
\begin{aligned}
u_{\varepsilon}\left(y_{\varepsilon}\right)= & \int_{B\left(x_{\varepsilon}, r_{\varepsilon}\right)} \mathcal{G}_{\varepsilon, 1}\left(\Delta u_{\varepsilon}+h_{\varepsilon} u_{\varepsilon}\right) d x \\
& +O\left(r_{\varepsilon}^{-1} \int_{\partial B\left(x_{\varepsilon}, r_{\varepsilon}\right)}\left|\partial_{\nu} u_{\varepsilon}\right| d \sigma\right)+O\left(r_{\varepsilon}^{-2} \int_{\partial B\left(x_{\varepsilon}, r_{\varepsilon}\right)} u_{\varepsilon} d \sigma\right) .
\end{aligned}
$$

This gives, by (1.15), (1.16) and (1.18),

$$
u_{\varepsilon}\left(y_{\varepsilon}\right)=O\left(\int_{B\left(x_{\varepsilon}, r_{\varepsilon}\right)}\left|x-y_{\varepsilon}\right|^{-1}\left|\Delta u_{\varepsilon}+h_{\varepsilon} u_{\varepsilon}\right| d x\right)+O\left(\alpha_{\varepsilon}\right) .
$$

Using 1.14 with $v=1 / 5$, we can write

$$
\begin{aligned}
& \int_{B\left(x_{\varepsilon}, r_{\varepsilon}\right)}\left|x-y_{\varepsilon}\right|^{-1}\left|\Delta u_{\varepsilon}+h_{\varepsilon} u_{\varepsilon}\right| d x \\
&=\int_{B\left(x_{\varepsilon}, \mu_{\varepsilon}\right)} \frac{u_{\varepsilon}^{5}}{\left|x-y_{\varepsilon}\right|} d x+\int_{B\left(x_{\varepsilon}, r_{\varepsilon}\right) \backslash B\left(x_{\varepsilon}, \mu_{\varepsilon}\right)}\left|x-y_{\varepsilon}\right|^{-1} u_{\varepsilon}^{5} d x \\
&= O\left(\mu_{\varepsilon}^{1 / 2}\left|y_{\varepsilon}-x_{\varepsilon}\right|^{-1}\right)+\alpha_{\varepsilon}^{5} r_{\varepsilon} \int_{B\left(x_{\varepsilon}, r_{\varepsilon}\right) \backslash B\left(x_{\varepsilon}, \mu_{\varepsilon}\right)}\left|x-y_{\varepsilon}\right|^{-1}\left|x-x_{\varepsilon}\right|^{-1} d x \\
&+\mu_{\varepsilon}^{3 / 2} \int_{B\left(x_{\varepsilon}, r_{\varepsilon}\right) \backslash B\left(x_{\varepsilon}, \mu_{\varepsilon}\right)}\left|x-y_{\varepsilon}\right|^{-1}\left|x-x_{\varepsilon}\right|^{-4} d x \\
&= O\left(\mu_{\varepsilon}^{1 / 2}\left|y_{\varepsilon}-x_{\varepsilon}\right|^{-1}\right)+O\left(\alpha_{\varepsilon}^{5} r_{\varepsilon}^{2}\right) .
\end{aligned}
$$

Thanks to 1.13 and (1.17), this leads to

$$
\int_{B\left(x_{\varepsilon}, r_{\varepsilon}\right)}\left|x-y_{\varepsilon}\right|^{-1}\left|\Delta u_{\varepsilon}\right| d x \leq O\left(\mu_{\varepsilon}^{1 / 2}\left|y_{\varepsilon}-x_{\varepsilon}\right|^{-1}\right)+o\left(\alpha_{\varepsilon}\right),
$$

which, by 1.22, proves (1.21).

In order to end the proof of the first part of the claim, we just have to show that

$$
\alpha_{\varepsilon}=\sup _{\partial B\left(x_{\varepsilon}, r_{\varepsilon}\right)} u_{\varepsilon}=O\left(\mu_{\varepsilon}^{1 / 2} r_{\varepsilon}^{-1}\right)
$$


For that purpose, we use the definition of $r_{\varepsilon}$ to find that

$$
\left(\beta r_{\varepsilon}\right)^{1 / 2} \psi_{\varepsilon}\left(\beta r_{\varepsilon}\right) \geq r_{\varepsilon}^{1 / 2} \psi_{\varepsilon}\left(r_{\varepsilon}\right)
$$

for all $0<\beta<1$. Using (1.16), this leads to

$$
r_{\varepsilon}^{1 / 2} \sup _{\partial B\left(x_{\varepsilon}, r_{\varepsilon}\right)} u_{\varepsilon} \leq C\left(\beta r_{\varepsilon}\right)^{1 / 2} \sup _{\partial B\left(x_{\varepsilon}, \beta r_{\varepsilon}\right)} u_{\varepsilon}
$$

From 1.21, we obtain

$$
\sup _{\partial B\left(x_{\varepsilon}, r_{\varepsilon}\right)} u_{\varepsilon} \leq C \beta^{1 / 2}\left(\mu_{\varepsilon}^{1 / 2}\left(\beta r_{\varepsilon}\right)^{-1}+\sup _{\partial B\left(x_{\varepsilon}, r_{\varepsilon}\right)} u_{\varepsilon}\right) .
$$

Choosing $\beta$ small enough clearly gives 1.23 and thus the pointwise estimate on $u_{\varepsilon}$ of the claim. The estimate on $\nabla u_{\varepsilon}$ then follows from standard elliptic theory.

We now prove the following:

Claim 1.3. If $r_{\varepsilon} \rightarrow 0$ as $\varepsilon \rightarrow 0$, then up to passing to a subsequence,

$$
r_{\varepsilon} u_{\varepsilon}\left(x_{\varepsilon}\right) u_{\varepsilon}\left(x_{\varepsilon}+r_{\varepsilon} x\right) \rightarrow \frac{1}{|x|}+b \quad \text { in } C_{\mathrm{loc}}^{1}(B(0,2) \backslash\{0\}) \text { as } \varepsilon \rightarrow 0
$$

where $b$ is some harmonic function in $B(0,2)$. Moreover, if $r_{\varepsilon}<\rho_{\varepsilon}$, then $b(0)=1$.

Proof. We set, for $x \in B(0,2)$,

$$
\tilde{u}_{\varepsilon}(x)=\mu_{\varepsilon}^{-1 / 2} r_{\varepsilon} u_{\varepsilon}\left(x_{\varepsilon}+r_{\varepsilon} x\right),
$$

which satisfies

$$
\Delta \tilde{u}_{\varepsilon}+r_{\varepsilon}^{2} \tilde{h}_{\varepsilon} \tilde{u}_{\varepsilon}=\left(\mu_{\varepsilon} / r_{\varepsilon}\right)^{2} \tilde{u}_{\varepsilon}^{5} \quad \text { in } B(0,2)
$$

where $\tilde{h}_{\varepsilon}=h\left(x_{\varepsilon}+r_{\varepsilon} x\right)$. Thanks to Claim 1.2, there exists $C>0$ such that

$$
\tilde{u}_{\varepsilon}(x) \leq C /|x| \quad \text { in } B(0,2) \backslash\{0\} .
$$

Then, by standard elliptic theory, after passing to a subsequence, we have $\tilde{u}_{\varepsilon} \rightarrow U$ in $C_{\text {loc }}^{1}(B(0,2) \backslash\{0\})$ as $\varepsilon \rightarrow 0$ where $U$ is a non-negative solution of

$$
\Delta U=0 \quad \text { in } B(0,2) \backslash\{0\} .
$$

Then, thanks to the Bôcher theorem on singularities of harmonic functions, we get

$$
U(x)=\lambda /|x|+b(x)
$$

where $b$ is some harmonic function in $B(0,2)$ and $\lambda \geq 0$. Now, integrating (1.24) on $B(0,1)$, we get

$$
\int_{\partial B(0,1)} \partial_{\nu} \tilde{u}_{\varepsilon} d \sigma=\int_{B(0,1)}\left(r_{\varepsilon}^{2} \tilde{h}_{\varepsilon} \tilde{u}_{\varepsilon}-\left(\mu_{\varepsilon} / r_{\varepsilon}\right)^{2} \tilde{u}_{\varepsilon}^{5}\right) d x .
$$


Thanks to Claim 1.2

$$
\int_{B(0,1)} r_{\varepsilon}^{2} \tilde{h}_{\varepsilon} \tilde{u}_{\varepsilon} d x \rightarrow 0 \quad \text { as } \varepsilon \rightarrow 0
$$

and, by Claim 1.1

$$
\int_{B(0,1)}\left(\mu_{\varepsilon} / r_{\varepsilon}\right)^{2} \tilde{u}_{\varepsilon}^{5} d x \rightarrow \int_{\mathbb{R}^{3}}\left(1+|x|^{2} / 3\right)^{-5 / 2} d x=\omega_{2} \quad \text { as } \varepsilon \rightarrow 0 .
$$

On the other hand,

$$
\int_{\partial B(0,1)} \partial_{\nu} \tilde{u}_{\varepsilon} d \sigma \rightarrow-\omega_{2} \lambda \quad \text { as } \varepsilon \rightarrow 0 .
$$

We deduce that $\lambda=1$, which proves the first part of the claim.

Now, if $r_{\varepsilon}<\rho_{\varepsilon}$, from the definition of $r_{\varepsilon}$ we have

$$
\psi_{\varepsilon}^{\prime}\left(r_{\varepsilon}\right)=0
$$

Setting $\tilde{\psi}_{\varepsilon}(r)=\left(r_{\varepsilon} / \mu_{\varepsilon}\right)^{1 / 2} \psi_{\varepsilon}\left(r_{\varepsilon} r\right)$ for $0<r<2$, we see that

$$
\tilde{\psi}_{\varepsilon}(r) \rightarrow \frac{r^{1 / 2}}{\omega_{2} r^{2}} \int_{\partial B(0, r)} U d \sigma=r^{-1 / 2}+r^{1 / 2} b(0) .
$$

We deduce that $b(0)=1$, which ends the proof of the claim.

We prove at last the following:

Claim 1.4. Using the notations of Claim 1.3 we have $b(0)=0$, and if the convergence in 1.2 holds in $C^{0, \eta}$, then $\nabla b(0)=0$.

Proof. We use the notation of the proof of Claim 1.3. Let us apply the Pohožaev identity (4.4) of Appendix 4.3 to $\tilde{u}_{\varepsilon}$ in $B(0,1)$. We obtain

$$
\frac{1}{2} \int_{B(0,1)} r_{\varepsilon}^{2}\left(\tilde{h}_{\varepsilon} \tilde{u}_{\varepsilon}^{2}+\tilde{h}_{\varepsilon}\left\langle x, \nabla \tilde{u}_{\varepsilon}^{2}\right\rangle\right) d x=\tilde{B}_{1}^{\varepsilon}+\tilde{B}_{2}^{\varepsilon}
$$

where

$$
\tilde{B}_{1}^{\varepsilon}=\int_{\partial B(0,1)}\left(\left(\partial_{\nu} \tilde{u}_{\varepsilon}\right)^{2}+\frac{1}{2} \tilde{u}_{\varepsilon} \partial_{\nu} \tilde{u}_{\varepsilon}-\frac{\left|\nabla \tilde{u}_{\varepsilon}\right|^{2}}{2}\right) d \sigma, \quad \tilde{B}_{2}^{\varepsilon}=\int_{\partial B(0,1)}\left(\frac{\mu_{\varepsilon}}{r_{\varepsilon}}\right)^{2} \frac{\tilde{u}_{\varepsilon}^{6}}{6} d \sigma .
$$

By Claim 1.2 and Lebesgue's dominated convergence theorem, we can pass to the limit to obtain

$$
\int_{\partial B(0,1)}\left(\left(\partial_{\nu} U\right)^{2}+\frac{1}{2} U \partial_{\nu} U-\frac{|\nabla U|^{2}}{2}\right) d \sigma=0 .
$$

Since $b$ is harmonic, it is easily checked that the left-hand side is just $-\omega_{2} b(0) / 2$. This proves that $b(0)=0$. 
In order to prove the second part of the claim, we apply the Pohožaev identity 4.7 of Appendix 4.3 to $\tilde{u}_{\varepsilon}$ in $B(0,1)$. We obtain

$$
\int_{\partial B(0,1)}\left(\frac{\left|\nabla \tilde{u}_{\varepsilon}\right|^{2}}{2} v-\partial_{\nu} \tilde{u}_{\varepsilon} \nabla \tilde{u}_{\varepsilon}\right) d \sigma=-\int_{B(0,1)} r_{\varepsilon}^{2} \tilde{h}_{\varepsilon} \frac{\nabla \tilde{u}_{\varepsilon}^{2}}{2} d x-\int_{\partial B(0,1)}\left(\frac{\mu_{\varepsilon}}{r_{\varepsilon}}\right)^{2} \frac{\tilde{u}_{\varepsilon}^{6}}{6} v d \sigma .
$$

It is clear that

$$
\int_{\partial B(0,1)}\left(\frac{\left|\nabla \tilde{u}_{\varepsilon}\right|^{2}}{2} v-\partial_{\nu} \tilde{u}_{\varepsilon} \nabla \tilde{u}_{\varepsilon}\right) d \sigma \rightarrow \int_{\partial B(0,1)}\left(\frac{|\nabla U|^{2}}{2} v-\partial_{\nu} U \nabla U\right) d \sigma \quad \text { as } \varepsilon \rightarrow 0 .
$$

Moreover, as $b$ is harmonic, we easily get

$$
\int_{\partial B(0,1)}\left(\frac{|\nabla U|^{2}}{2} v-\nabla U \partial_{\nu} U\right) d \sigma=\omega_{2} \nabla b(0) .
$$

It remains to deal with the right-hand side of 1.26 . It is clear that

$$
\int_{\partial B(0,1)}\left(\frac{\mu_{\varepsilon}}{r_{\varepsilon}}\right)^{2} \frac{\tilde{u}_{\varepsilon}^{6}}{6} v d \sigma \rightarrow 0 \quad \text { as } \varepsilon \rightarrow 0
$$

Then we rewrite the first term of the right-hand side of (1.26) as

$$
\int_{B(0,1)} r_{\varepsilon}^{2} \tilde{h}_{\varepsilon} \frac{\nabla \tilde{u}_{\varepsilon}^{2}}{2} d x=\int_{B(0,1)} r_{\varepsilon}^{2}\left(\tilde{h}_{\varepsilon}-\tilde{h}_{\varepsilon}(0)\right) \frac{\nabla \tilde{u}_{\varepsilon}^{2}}{2} d x+\tilde{h}_{\varepsilon}(0) \int_{B(0,1)} r_{\varepsilon}^{2} \frac{\nabla \tilde{u}_{\varepsilon}^{2}}{2} d x
$$

If we assume that the convergence of $\left(h_{\varepsilon}\right)$ holds in $C^{0, \eta}$, we can use Lebesgue's dominated convergence theorem to conclude that the first term of the right-hand side goes to 0 as $\varepsilon \rightarrow 0$. Then, integrating the second term by parts, we get

$$
\tilde{h}_{\varepsilon}(0) \int_{B(0,1)} r_{\varepsilon}^{2} \frac{\nabla \tilde{u}_{\varepsilon}^{2}}{2} d x=\tilde{h}_{\varepsilon}(0) \int_{\partial B(0,1)} r_{\varepsilon}^{2} \frac{\tilde{u}_{\varepsilon}^{2}}{2} \nu d \sigma
$$

which clearly goes to 0 as $\varepsilon \rightarrow 0$. Finally, collecting the above information, and passing to the limit $\varepsilon \rightarrow 0$ in 1.26 , we get $\nabla b(0)=0$ if the convergence of $\left(h_{\varepsilon}\right)$ holds in $C^{0, \eta}$, which completes the proof of the claim.

We are now in a position to end the proof of Proposition 1.1. If $\rho_{\varepsilon} \rightarrow 0$ as $\varepsilon \rightarrow 0$ then we deduce the proposition from Claims 1.3 and 1.4. If $\rho_{\varepsilon} \nrightarrow 0$ as $\varepsilon \rightarrow 0$, then Claims 1.3 and 1.4 give $r_{\varepsilon} \nrightarrow 0$ as $\varepsilon \rightarrow 0$. Then, using the Harnack inequality (1.16, one can extend the result of Claim 1.2 to $B\left(x_{\varepsilon}, 2 \rho_{\varepsilon}\right) \backslash\left\{x_{\varepsilon}\right\}$, which proves the first part of Proposition 1.1 when $\rho_{\varepsilon} \nrightarrow 0$, and ends the proof of the whole proposition. 


\section{Stability of the Pohožaev obstruction}

We now prove Theorem 1 and give some stability result for radial solutions on the unit ball (see end of the section). We assume by contradiction that there exists a sequence $\left(h_{\varepsilon}\right)$ of functions in $C^{0, \eta}\left(\mathbb{R}^{3}\right)$ for some $\eta>0$ and a sequence $\left(u_{\varepsilon}\right)$ of $C^{2}$-solutions of 1.1 where $\Omega$ is some smooth domain in $\mathbb{R}^{3}$ star-shaped with respect to the origin and $h_{\varepsilon} \rightarrow h$ in $L^{p}(\Omega)$ as $\varepsilon \rightarrow 0$ for some $p>3$ where $h \in C^{1}\left(\mathbb{R}^{3}\right)$ satisfies 0.2 . Sometimes we will assume that $h_{\varepsilon} \rightarrow h$ in $C^{0, \eta}$ as $\varepsilon \rightarrow 0$.

We claim first that

$$
\left\|u_{\varepsilon}\right\|_{\infty} \rightarrow+\infty \quad \text { as } \varepsilon \rightarrow 0 .
$$

Indeed, if $\left(u_{\varepsilon}\right)$ is uniformly bounded in $L^{\infty}(\Omega)$, then it is clear that $\left(u_{\varepsilon} /\left\|u_{\varepsilon}\right\|_{\infty}\right)$ is uniformly bounded in $W^{2, p}(\Omega)$ for some $p>3$, and thus, after passing to a subsequence, $u_{\varepsilon} /\left\|u_{\varepsilon}\right\|_{\infty} \rightarrow u$ in $C_{\text {loc }}^{1}(\Omega)$ where $u$ is a positive solution of

$$
\Delta u+h u=\left(\lim _{\varepsilon \rightarrow 0}\left\|u_{\varepsilon}\right\|_{\infty}^{4}\right) u^{5} \quad \text { in } \Omega
$$

with $u=0$ on $\partial \Omega$. Since $h \geq 0$, it is clear that $\left\|u_{\varepsilon}\right\|_{\infty} \nrightarrow 0$ as $\varepsilon \rightarrow 0$. Then $\tilde{u}=$ $\left(\lim _{\varepsilon \rightarrow 0}\left\|u_{\varepsilon}\right\|_{\infty}\right) u$ is a non-trivial solution of $(0.1)$, which is a contradiction since $(0.2)$ holds. Thus 2.1) is proved.

Then the sequence $\left(u_{\varepsilon}\right)$ develops some concentration phenomena. We prove that this leads to a contradiction as follows: in Claim 2.1, mimicking [8], we construct a family of critical points of $u_{\varepsilon},\left(x_{1, \varepsilon}, \ldots, x_{N_{\varepsilon}, \varepsilon}\right)$, such that each sequence $\left(x_{i_{\varepsilon}, \varepsilon}\right)$ satisfies the assumptions of Section 1 with

$$
\rho_{\varepsilon}=\min _{1 \leq i \leq N_{\varepsilon}, i \neq i_{\varepsilon}}\left\{\left|x_{i, \varepsilon}-x_{i_{\varepsilon}, \varepsilon}\right|, d\left(x_{i_{\varepsilon}, \varepsilon}, \partial \Omega\right)\right\}
$$

In Claim 2.2, we prove that these concentration points are in fact isolated. In other words, we prove that $\left(u_{\varepsilon}\right)$ develops only finitely many concentration points. We prove that such a configuration of concentration points must satisfy two relations involving the Green function of $\Delta+h$ at these points. And it is impossible to find such a configuration thanks to some Pohožaev identity on Green functions we prove in Appendix 4.4. Claim 2.1 is rather classical. The core of the proof lies in Claim 2.2. Avoiding bubble accumulation in the interior of $\Omega$ in dimension 3 is by now classical. The main difficulty here is to avoid boundary bubble accumulation. The rest of the section is devoted to the details of the proof we have just sketched.

Claim 2.1. There exists $D>0$ such that for all $\varepsilon>0$, there exists $N_{\varepsilon} \in \mathbb{N}^{*}$ and $N_{\varepsilon}$ critical points of $u_{\varepsilon}$, denoted by $\left(x_{1, \varepsilon}, \ldots, x_{N_{\varepsilon}, \varepsilon}\right)$, such that

$$
\begin{array}{ll}
d\left(x_{i, \varepsilon}, \partial \Omega\right) u_{\varepsilon}\left(x_{i, \varepsilon}\right)^{2} \geq 1 & \text { for all } i \in\left[1, N_{\varepsilon}\right], \\
\left|x_{i, \varepsilon}-x_{j, \varepsilon}\right| u_{\varepsilon}\left(x_{i, \varepsilon}\right)^{2} \geq 1 & \text { for all } i \neq j \in\left[1, N_{\varepsilon}\right],
\end{array}
$$

and

$$
\min _{i \in\left[1, N_{\varepsilon}\right]}\left|x_{i, \varepsilon}-x\right| u_{\varepsilon}(x)^{2} \leq D \quad \text { for all } x \in \Omega \text { and all } \varepsilon>0
$$


Proof. First of all, we claim that

$$
\left\{x \in \Omega: \nabla u_{\varepsilon}(x)=0 \text { and } d(x, \partial \Omega) u_{\varepsilon}(x)^{2} \geq 1\right\} \neq \varnothing
$$

for $\varepsilon$ small enough. Let us prove 2.2). Let $y_{\varepsilon} \in \Omega$ be a point where $u_{\varepsilon}$ achieves its maximum. We set $\mu_{\varepsilon}=u_{\varepsilon}\left(y_{\varepsilon}\right)^{-2} \rightarrow 0$ as $\varepsilon \rightarrow 0$. We set also, for all $x \in \Omega_{\varepsilon}=$ $\left\{x \in \mathbb{R}^{3}: y_{\varepsilon}+\mu_{\varepsilon} x \in \Omega\right\}$,

$$
\tilde{u}_{\varepsilon}(x)=\mu_{\varepsilon}^{1 / 2} u_{\varepsilon}\left(y_{\varepsilon}+\mu_{\varepsilon} x\right)
$$

which satisfies

$$
\Delta \tilde{u}_{\varepsilon}+\mu_{\varepsilon}^{2} \tilde{h}_{\varepsilon} \tilde{u}_{\varepsilon}=\tilde{u}_{\varepsilon}^{5} \quad \text { in } \Omega_{\varepsilon}
$$

where $\tilde{h}_{\varepsilon}=h\left(y_{\varepsilon}+\mu_{\varepsilon} x\right)$. Note that $0 \leq \tilde{u}_{\varepsilon} \leq \tilde{u}_{\varepsilon}(0)=1$. By standard elliptic theory, $\tilde{u}_{\varepsilon} \rightarrow U$ in $C_{\text {loc }}^{1}\left(\Omega_{0}\right)$ where $U$ satisfies

$$
\Delta U=U^{5} \quad \text { in } \Omega_{0} \quad \text { and } \quad 0 \leq U \leq 1=U(0),
$$

and where $\Omega_{0}=\lim _{\varepsilon \rightarrow 0} \Omega_{\varepsilon}$. Thanks to [5], we have $\Omega_{0}=\mathbb{R}^{3}$, which proves that $d\left(y_{\varepsilon}, \partial \Omega\right) u_{\varepsilon}\left(y_{\varepsilon}\right)^{2} \rightarrow+\infty$ as $\varepsilon \rightarrow 0$. This ends the proof of 2.2.

Now, in view of Lemma 4.1 (see Appendix 4.1), for $\varepsilon$ small enough, there exist $N_{\varepsilon} \in \mathbb{N}^{*}$ and $N_{\varepsilon}$ critical points of $u_{\varepsilon}$, denoted by $\left(x_{1, \varepsilon}, \ldots, x_{N_{\varepsilon}, \varepsilon}\right)$, such that

$$
\begin{array}{ll}
d\left(x_{i, \varepsilon}, \partial \Omega\right) u_{\varepsilon}\left(x_{i, \varepsilon}\right)^{2} \geq 1 & \text { for all } i \in\left[1, N_{\varepsilon}\right], \\
\left|x_{i, \varepsilon}-x_{j, \varepsilon}\right| u_{\varepsilon}\left(x_{i, \varepsilon}\right)^{2} \geq 1 & \text { for all } i \neq j \in\left[1, N_{\varepsilon}\right],
\end{array}
$$

and

$$
\min _{i \in\left[1, N_{\varepsilon}\right]}\left|x_{i, \varepsilon}-x\right| u_{\varepsilon}(x)^{2} \leq 1
$$

for all critical points $x$ of $u_{\varepsilon}$ such that $d(x, \partial \Omega) u_{\varepsilon}(x)^{2} \geq 1$. It remains to show that there exists $D>0$ such that

$$
\min _{i \in\left[1, N_{\varepsilon}\right]}\left|x_{i, \varepsilon}-x\right| u_{\varepsilon}(x)^{2} \leq D
$$

for all $x \in \Omega$. We proceed by contradiction, assuming that

$$
\sup _{x \in \Omega} \min _{i \in\left[1, N_{\varepsilon}\right]}\left|x_{i, \varepsilon}-x\right| u_{\varepsilon}(x)^{2} \rightarrow+\infty
$$

as $\varepsilon \rightarrow 0$. Let $z_{\varepsilon} \in \Omega$ be such that

$$
\min _{i \in\left[1, N_{\varepsilon}\right]}\left|x_{i, \varepsilon}-z_{\varepsilon}\right| u_{\varepsilon}\left(z_{\varepsilon}\right)^{2}=\sup _{x \in \Omega} \min _{i \in\left[1, N_{\varepsilon}\right]}\left|x_{i, \varepsilon}-x\right| u_{\varepsilon}(x)^{2} .
$$

We set $\hat{\mu}_{\varepsilon}=u_{\varepsilon}\left(z_{\varepsilon}\right)^{-2}$ and $S_{\varepsilon}=\left\{x_{1, \varepsilon}, \ldots, x_{N_{\varepsilon}, \varepsilon}\right\}$. Thanks to 2.4, we find that $\hat{\mu}_{\varepsilon} \rightarrow 0$ as $\varepsilon \rightarrow 0$ and

$$
d\left(S_{\varepsilon}, z_{\varepsilon}\right) / \hat{\mu}_{\varepsilon} \rightarrow+\infty \quad \text { as } \varepsilon \rightarrow 0 .
$$

Then we set, for all $x \in \hat{\Omega}_{\varepsilon}=\left\{x \in \mathbb{R}^{3}: z_{\varepsilon}+\hat{\mu}_{\varepsilon} x \in \Omega\right\}$,

$$
\hat{u}_{\varepsilon}(x)=\hat{\mu}_{\varepsilon}^{1 / 2} \hat{u}_{\varepsilon}\left(z_{\varepsilon}+\hat{\mu}_{\varepsilon} x\right),
$$


which satisfies

$$
\Delta \hat{u}_{\varepsilon}+\hat{\mu}_{\varepsilon}^{2} \hat{h}_{\varepsilon} \hat{u}_{\varepsilon}=\hat{u}_{\varepsilon}^{5} \quad \text { in } \Omega_{\varepsilon}
$$

where $\hat{h}_{\varepsilon}=h\left(z_{\varepsilon}+\hat{\mu}_{\varepsilon} x\right)$. Note that $\hat{u}_{\varepsilon}(0)=1$ and also

$$
\lim _{\varepsilon \rightarrow 0} \sup _{B(0, R) \cap \Omega_{\varepsilon}} \hat{u}_{\varepsilon}=1
$$

for all $R>0$ thanks to 2.4 and 2.5. Standard elliptic theory then gives $\hat{u}_{\varepsilon} \rightarrow \hat{U}$ in $C_{\text {loc }}^{1}\left(\hat{\Omega}_{0}\right)$ where $U$ satisfies

$$
\Delta \hat{U}=\hat{U}^{5} \quad \text { in } \hat{\Omega}_{0} \quad \text { and } \quad 0 \leq \hat{U} \leq 1=\hat{U}(0)
$$

with $\hat{\Omega}_{0}=\lim _{\varepsilon \rightarrow 0} \hat{\Omega}_{\varepsilon}$. As above, we deduce that $\hat{\Omega}_{0}=\mathbb{R}^{3}$, which gives

$$
\lim _{\varepsilon \rightarrow 0} d\left(z_{\varepsilon}, \partial \Omega\right) u_{\varepsilon}^{2}\left(z_{\varepsilon}\right)=+\infty
$$

Moreover, thanks to [4], we know that

$$
\hat{U}(x)=\frac{1}{\left(1+|x|^{2} / 3\right)^{1 / 2}} .
$$

Since $\hat{U}$ has a strict local maximum at 0 , there exists $\hat{x}_{\varepsilon}$, a critical point of $u_{\varepsilon}$, such that $\left|z_{\varepsilon}-\hat{x}_{\varepsilon}\right|=o\left(\hat{\mu}_{\varepsilon}\right)$ and $\hat{\mu}_{\varepsilon} u_{\varepsilon}\left(\hat{x}_{\varepsilon}\right)^{2} \rightarrow 1$ as $\varepsilon \rightarrow 0$. Thanks to $(2.5)$ and $(2.6)$, this contradicts 2.3 and proves the claim.

We define

$$
d_{\varepsilon}=\min \left\{d\left(x_{i, \varepsilon}, x_{j, \varepsilon}\right), d\left(x_{i, \varepsilon}, \partial \Omega\right): 1 \leq i<j \leq N_{\varepsilon}\right\}
$$

and prove:

Claim 2.2. If the convergence of $h_{\varepsilon}$ to $h$ holds in $C^{0, \eta}$, then there exists $d>0$ such that $d_{\varepsilon} \geq d$.

Proof. Assume that $d_{\varepsilon} \rightarrow 0$ as $\varepsilon \rightarrow 0$. There are two cases to consider: either the distance between two critical points goes to 0 , or one of them goes to the boundary. In the first case, the arguments which lead to a contradiction follow closely [7], but in the second case we have to be more precise looking at the "artificial" singularities created by the boundary.

Up to reordering the concentration points, we can assume that

$$
d_{\varepsilon}=d\left(x_{1, \varepsilon}, x_{2, \varepsilon}\right) \text { or } d\left(x_{1, \varepsilon}, \partial \Omega\right) .
$$

For $x \in \Omega_{\varepsilon}=\left\{x \in \mathbb{R}^{3}: x_{1, \varepsilon}+d_{\varepsilon} x \in \Omega\right\}$, we set

$$
\tilde{u}_{\varepsilon}(x)=d_{\varepsilon}^{1 / 2} u_{\varepsilon}\left(x_{1, \varepsilon}+d_{\varepsilon} x\right),
$$

which satisfies

$$
\Delta \tilde{u}_{\varepsilon}+d_{\varepsilon}^{2} \tilde{h}_{\varepsilon} \tilde{u}_{\varepsilon}=\tilde{u}_{\varepsilon}^{5} \quad \text { in } \Omega_{\varepsilon}
$$


where $\tilde{h}_{\varepsilon}=h\left(x_{1, \varepsilon}+d_{\varepsilon} x\right)$. We have, up to a harmless rotation,

$$
\left.\lim _{\varepsilon \rightarrow 0} \Omega_{\varepsilon}=\Omega_{0}=\mathbb{R}^{3} \text { or }\right]-\infty, d\left[\times \mathbb{R}^{2} \text { where } d \geq 1 .\right.
$$

We also set

$$
\tilde{x}_{i, \varepsilon}=\frac{x_{i, \varepsilon}-x_{1, \varepsilon}}{d_{\varepsilon}} .
$$

We claim that, for any sequence $i_{\varepsilon} \in\left[1, N_{\varepsilon}\right]$ such that

$$
\tilde{u}_{\varepsilon}\left(\tilde{x}_{i_{\varepsilon}, \varepsilon}\right)=O(1)
$$

we have

$$
\sup _{B\left(\tilde{x}_{i \varepsilon, \varepsilon}, 1 / 2\right)} \tilde{u}_{\varepsilon}=O(1)
$$

Indeed, let $y_{\varepsilon} \in \overline{B\left(\tilde{x}_{\varepsilon_{\varepsilon}, \varepsilon}, 1 / 2\right)}$ be such that $\sup _{B\left(\tilde{x}_{\varepsilon, \varepsilon}, 1 / 2\right)} \tilde{u}_{\varepsilon}=\tilde{u}_{\varepsilon}\left(y_{\varepsilon}\right)$ and assume by contradiction that

$$
\tilde{u}_{\varepsilon}\left(y_{\varepsilon}\right)^{2} \rightarrow+\infty \quad \text { as } \varepsilon \rightarrow 0 .
$$

Thanks to the definitions of $d_{\varepsilon}, y_{\varepsilon}$ and the last assertion of Claim 2.1.

$$
\left|d_{\varepsilon}\left(y_{\varepsilon}-\tilde{x}_{i_{\varepsilon}, \varepsilon}\right)\right| u_{\varepsilon}\left(x_{1, \varepsilon}+d_{\varepsilon} y_{\varepsilon}\right)^{2} \leq D
$$

so that

$$
\left|y_{\varepsilon}-\tilde{x}_{i_{\varepsilon}, \varepsilon}\right|=o(1)
$$

For $x \in B\left(0,1 /\left(3 \hat{\mu}_{\varepsilon}\right)\right)$ and $\varepsilon$ small enough, we set

$$
\hat{u}_{\varepsilon}(x)=\hat{\mu}_{\varepsilon}^{1 / 2} \tilde{u}_{\varepsilon}\left(y_{\varepsilon}+\hat{\mu}_{\varepsilon} x\right)
$$

where $\hat{\mu}_{\varepsilon}=u_{\varepsilon}\left(y_{\varepsilon}\right)^{-2}$. It satisfies

$$
\Delta \hat{u}_{\varepsilon}+\left(\hat{\mu}_{\varepsilon} d_{\varepsilon}\right)^{2} \hat{h}_{\varepsilon} \hat{u}_{\varepsilon}=\hat{u}_{\varepsilon}^{5} \quad \text { in } B\left(0, \frac{1}{3 \hat{\mu}_{\varepsilon}}\right) \quad \text { and } \quad \hat{u}_{\varepsilon}(0)=\sup _{B\left(0,1 /\left(3 \hat{\mu}_{\varepsilon}\right)\right)} \hat{u}_{\varepsilon}=1
$$

where $\hat{h}_{\varepsilon}=\tilde{h}_{\varepsilon}\left(y_{\varepsilon}+\hat{\mu}_{\varepsilon} x\right)$. Thanks to 2.9 , $B\left(0,1 /\left(3 \hat{\mu}_{\varepsilon}\right)\right) \rightarrow \mathbb{R}^{3}$ as $\varepsilon \rightarrow+\infty$. Then $\left(\hat{u}_{\varepsilon}\right)$ is locally uniformly bounded and, by standard elliptic theory, $\hat{u}_{\varepsilon}$ converges to $\hat{U}$ in $C_{\text {loc }}^{1}\left(\mathbb{R}^{3}\right)$ where $\hat{U}$ satisfies

$$
\Delta \hat{U}=\hat{U}^{5} \quad \text { in } \mathbb{R}^{3} \quad \text { and } \quad 0 \leq \hat{U} \leq 1=\hat{U}(0) .
$$

By [4] and the fact that $\left(\tilde{x}_{i_{\varepsilon}, \varepsilon}-y_{\varepsilon}\right) / \hat{\mu}_{\varepsilon}$ is bounded, we find that

$$
\liminf _{\varepsilon \rightarrow 0} \frac{\tilde{u}_{\varepsilon}\left(x_{i_{\varepsilon}, \varepsilon}\right)}{\tilde{u}_{\varepsilon}\left(y_{\varepsilon}\right)}>0,
$$

which is in contradiction with 2.7] and (2.9), and completes the proof of 2.8]. 
For $R>0$, we set $S_{R, \varepsilon}=\left\{\tilde{x}_{i, \varepsilon}: \tilde{x}_{i, \varepsilon} \in B(0, R)\right\}$. By the definition of $d_{\varepsilon}$, up to a subsequence, $S_{R, \varepsilon} \rightarrow S_{R}$ as $\varepsilon \rightarrow 0$, where $S_{R}$ is a non-empty finite set; then up to taking a diagonal subsequence, we can define the countable set

$$
S=\bigcup_{R>0} S_{R}
$$

Thanks to the previous definition, we are ready to prove the following assertion:

$$
\forall i_{\varepsilon} \in\left[1, N_{\varepsilon}\right] \text { with } d\left(x_{i_{\varepsilon}, \varepsilon}, x_{1, \varepsilon}\right)=O\left(d_{\varepsilon}\right), \quad \tilde{u}_{\varepsilon}\left(\tilde{x}_{i_{\varepsilon}, \varepsilon}\right) \rightarrow+\infty \quad \text { as } \varepsilon \rightarrow 0 .
$$

Assume that there exists $i_{\varepsilon}$ such that $d\left(x_{i_{\varepsilon}, \varepsilon}, x_{1, \varepsilon}\right)=O\left(d_{\varepsilon}\right)$ with $\tilde{u}_{\varepsilon}\left(\tilde{x}_{i_{\varepsilon}, \varepsilon}\right)$ bounded. Then for all sequences $j_{\varepsilon}$ such that $d\left(x_{j_{\varepsilon}, \varepsilon}, x_{1, \varepsilon}\right)=O\left(d_{\varepsilon}\right), \tilde{u}_{\varepsilon}\left(\tilde{x}_{j_{\varepsilon}, \varepsilon}\right)$ is bounded. Indeed, if there exists a sequence $j_{\varepsilon}$ such that $d\left(x_{j_{\varepsilon}, \varepsilon}, x_{1, \varepsilon}\right)=O\left(d_{\varepsilon}\right)$ and $\tilde{u}_{\varepsilon}\left(\tilde{x}_{j_{\varepsilon}, \varepsilon}\right) \rightarrow+\infty$ as $\varepsilon \rightarrow 0$, then thanks to Claim 2.1. we can apply Proposition 1.1 with $x_{\varepsilon}=\tilde{x}_{j_{\varepsilon}, \varepsilon}$ and $\rho_{\varepsilon}=d_{\varepsilon} / 3$. We find that up to a subsequence, $\tilde{u}_{\varepsilon} \rightarrow 0$ in $C_{\text {loc }}^{1}(B(\tilde{x}, 2 / 3)) \backslash\{\tilde{x}\}$, where $\tilde{x}=\lim _{\varepsilon \rightarrow 0} \tilde{x}_{j_{\varepsilon}, \varepsilon}$. But $\left(\tilde{u}_{\varepsilon}\right)$ is uniformly bounded in $B(\tilde{y}, 1 / 2)$, where $\tilde{y}=\lim _{\varepsilon \rightarrow 0} \tilde{x}_{i_{\varepsilon}, \varepsilon}$. We thus deduce from Harnack's inequality that $\tilde{u}_{\varepsilon}\left(\tilde{x}_{i_{\varepsilon}, \varepsilon}\right) \rightarrow 0$ as $\varepsilon \rightarrow 0$, in contradiction with the first or the second assertion of Claim 2.1.

Thus we have proved that for all sequences $j_{\varepsilon}$ such that $d\left(x_{j_{\varepsilon}, \varepsilon}, x_{1, \varepsilon}\right)=O\left(d_{\varepsilon}\right)$, $\tilde{u}_{\varepsilon}\left(\tilde{x}_{j_{\varepsilon}, \varepsilon}\right)$ is bounded, which proves that $\left(\tilde{u}_{\varepsilon}\right)$ is uniformly bounded in a neighbourhood of any finite subset of $S$. But thanks to Claim 2.1. $\tilde{u}_{\varepsilon}$ is bounded in any compact subset of $\Omega_{0} \backslash S$. This clearly proves that $\tilde{u}_{\varepsilon}$ is uniformly bounded on any compact subset of $\Omega_{0}$. Then, by standard elliptic theory, $\tilde{u}_{\varepsilon} \rightarrow U$ in $C_{\text {loc }}^{1}\left(\Omega_{0}\right)$ as $\varepsilon \rightarrow 0$, where $U$ is a non-negative solution of

$$
\Delta U=U^{5} \quad \text { in } \Omega_{0} .
$$

But, thanks to the first or second assertion of Claim 2.1. we know that $U(0) \geq 1$, hence necessarily $\Omega_{0}=\mathbb{R}^{3}$, and thus $U$ possesses at least two critical points, namely 0 and $\check{x}_{2}=\lim _{\varepsilon \rightarrow 0} \check{x}_{2, \varepsilon}$. By [4], this is impossible. This ends the proof of [2.11].

We are now going to consider two cases, depending on $\Omega_{0}$.

Case 1: $\Omega_{0}=\mathbb{R}^{3}$. In this case, up to a subsequence, $d_{\varepsilon}=d\left(x_{1, \varepsilon}, x_{2, \varepsilon}\right)$ and $S=\left\{0, \tilde{x}_{2}=\right.$ $\left.\lim _{\varepsilon \rightarrow 0} \tilde{x}_{2, \varepsilon}, \ldots\right\}$ contains at least two points. Applying Proposition 1.1 with $x_{\varepsilon}=\tilde{x}_{i, \varepsilon}$ and $\rho_{\varepsilon}=d_{\varepsilon} / 3$, we obtain

$$
\tilde{u}_{\varepsilon}(0) \tilde{u}_{\varepsilon}(x) \rightarrow H=\frac{1}{|x|}+\frac{\lambda_{2}}{\left|x-\tilde{x}_{2}\right|}+\tilde{b} \quad \text { in } C_{\text {loc }}^{1}\left(\mathbb{R}^{3} \backslash S\right) \text { as } \varepsilon \rightarrow 0
$$

where $\tilde{b}$ is a harmonic function in $\Omega_{0} \backslash\left\{S \backslash\left\{0, \check{x}_{2}\right\}\right\}$, and $\lambda_{2}>0$. Moreover $\tilde{b}(0)=-\lambda_{2}$. We prove in the following that $\tilde{b}$ is non-negative, which will give a contradiction and end the study of this case. To check that $\tilde{b}$ is non-negative, for each positive number $r$, we rewrite $H$ as

$$
H=\sum_{\tilde{x}_{i} \in S \cap B(0, r)} \frac{\lambda_{i}}{\left|x-\tilde{x}_{i}\right|}+\hat{b}_{r},
$$

where $\lambda_{i}>0$. Then, taking $R>r$ large enough, we get $\hat{b}_{r}>-1 / r$ on $\partial B(0, R)$. Moreover, for any $\tilde{x}_{j} \in B(0, R) \backslash B(0, r)$, there exists a neighbourhood $V_{j, r}$ of $\tilde{x}_{j}$ such 
that $\hat{b}_{r}>0$ on $V_{j, r}$. Thanks to the maximum principle, $\hat{b}_{r}>-1 / r$ on $B(0, R)$. Since $\hat{b}_{r} \rightarrow \hat{b}$ on every compact set as $r \rightarrow+\infty$, we see that $H=\sum_{\tilde{x}_{i} \in S} \lambda_{i} /\left|x-\tilde{x}_{i}\right|+\hat{b}$ with $\hat{b} \geq 0$, which proves that $\tilde{b} \geq 0$. This is the contradiction we have been looking for, and this ends the proof of the claim in this first case.

Case 2: $\left.\Omega_{0}=\right]-\infty, d\left[\times \mathbb{R}^{2}\right.$. We still denote $S=\left\{0=\tilde{x}_{1}, \tilde{x}_{2}, \ldots\right\}$ and we apply Proposition 1.1 with $x_{\varepsilon}=x_{i, \varepsilon}$ and $\rho_{\varepsilon}=d_{\varepsilon} / 3$ to deduce that

$$
\tilde{u}_{\varepsilon}(0) \tilde{u}_{\varepsilon}(x) \rightarrow H=\sum_{\tilde{x}_{i} \in S} \frac{\lambda_{i}}{\left|x-\tilde{x}_{i}\right|}+\tilde{b} \quad \text { in } C_{\mathrm{loc}}^{1}\left(\Omega_{0} \backslash S\right)
$$

where $\lambda_{i}>0$ and $\tilde{b}$ is some harmonic function in $\Omega_{0}$. We extend $H$ to $\mathbb{R}^{3}$ by setting

$$
\hat{H}(x)= \begin{cases}H(x) & \text { if } x_{1} \leq d, \\ -H(s(x)) & \text { otherwise, }\end{cases}
$$

where $s$ is the symmetry with respect to $\{d\} \times \mathbb{R}^{2}$. We also extend $\tilde{b}$ by setting

$$
\hat{H}=\sum_{\tilde{x}_{i} \in S}\left(\frac{\lambda_{i}}{\left|x-\tilde{x}_{i}\right|}-\frac{\lambda_{i}}{\left|s(x)-\tilde{x}_{i}\right|}\right)+\hat{b} .
$$

It is clear that $\hat{b}$ is harmonic on $\mathbb{R}^{3}$ and satisfies $\hat{b} \geq 0$ in $\Omega_{0}$ and $\hat{b} \leq 0$ in $\mathbb{R}^{3} \backslash \Omega_{0}$. This can be proved as in Case 1. Let $\mathcal{G}_{R}$ be the Green function of the Laplacian on the ball centred at 0 with radius $R$. The Green representation formula yields

$$
\hat{b}(x)=\int_{\partial B(0, R)} \partial_{\nu} \mathcal{G}_{R}(x, y) \hat{b}(y) d \sigma
$$

since

$$
\partial_{\nu} \mathcal{G}_{R}(x, y)=\frac{R^{2}-|x|^{2}}{\omega_{2} R|x-y|^{3}}
$$

on $\partial B(0, R)$, this gives

$$
\partial_{1} \hat{b}(0)=\frac{3}{\omega_{2} R^{4}} \int_{\partial B(0, R)} y_{1} \hat{b}(y) d \sigma .
$$

Now we decompose $\partial B(0, R)$ into three sets:

$$
\begin{aligned}
& A=\left\{y \in \partial B(0, R): y_{1} \geq d\right\}, \\
& B=\left\{y \in \partial B(0, R): 0 \leq y_{1} \leq d\right\}, \\
& C=\left\{y \in \partial B(0, R): y_{1} \leq 0\right\} .
\end{aligned}
$$

In $A$ and $B$, we have $y_{1} \hat{b}(y) \leq d \hat{b}(y)$, and in $C$, we have $y_{1} \hat{b}(y) \leq 0$. Since $\hat{b} \geq 0$ in $C$, we arrive at

$$
\partial_{1} \hat{b}(0) \leq \frac{3 d}{\omega_{2} R^{4}} \int_{A \cup B} \hat{b}(y) d \sigma \leq \frac{3 d}{\omega_{2} R^{4}} \int_{\partial B(0, R)} \hat{b}(y) d \sigma=\frac{3 d \hat{b}(0)}{R^{2}} .
$$


Passing to the limit as $R \rightarrow+\infty$ gives $\partial_{1} \hat{b}(0) \leq 0$. In order to obtain a contradiction, we rewrite $H$ in a neighbourhood of 0 as

$$
H(x)=\frac{1}{|x|}+\check{b}(x)
$$

where

$$
\check{b}(x)=\hat{b}(x)-\frac{1}{|s(x)|}+\sum_{\check{x}_{i} \in S \backslash\{0\}} \lambda_{i}\left(\frac{1}{\left|x-\check{x}_{i}\right|}-\frac{1}{\left|s(x)-\check{x}_{i}\right|}\right) .
$$

As is easily checked, $\partial_{1} \breve{b}(0)<0$, contrary to Proposition 1.1 . This ends the proof of Claim 2.2 in this second case.

We are now ready to prove Theorem 1 Thanks to Claim 2.1. there exist $D>0$, $N \in \mathbb{N}^{*}$ and $N$ local maxima of $u_{\varepsilon}, x_{1, \varepsilon}, \ldots, x_{N, \varepsilon}$, such that

$$
\begin{array}{ll}
d\left(x_{i, \varepsilon}, \partial \Omega\right) u_{\varepsilon}\left(x_{i, \varepsilon}\right)^{2} \geq 1 & \text { for all } i \in[1, N], \\
\left|x_{i, \varepsilon}-x_{j, \varepsilon}\right| u_{\varepsilon}\left(x_{i, \varepsilon}\right)^{2} \geq 1 & \text { for all } i \neq j \in[1, N],
\end{array}
$$

and

$$
\min _{i \in[1, N]}\left|x_{i, \varepsilon}-x\right| u_{\varepsilon}(x)^{2} \leq D \quad \text { for all } x \in \Omega .
$$

We can assume that $u_{\varepsilon}\left(x_{i, \varepsilon}\right) \rightarrow+\infty$ as $\varepsilon \rightarrow 0$. Indeed, otherwise we can remove $x_{i, \varepsilon}$ from the family of concentration points, and up to changing $D$, the assertion remains true. Then, thanks to the Harnack inequality, there exists $C>0$ such that

$$
\frac{1}{C} u_{\varepsilon}\left(x_{1, \varepsilon}\right) \leq u_{\varepsilon}\left(x_{i, \varepsilon}\right) \leq C u_{\varepsilon}\left(x_{1, \varepsilon}\right) .
$$

Now, thanks to the results of Section 1 and by standard elliptic theory, we have, after passing to a subsequence,

$$
u_{\varepsilon}\left(x_{1, \varepsilon}\right) u_{\varepsilon}(x) \rightarrow G \quad \text { in } C_{\mathrm{loc}}^{2}\left(\Omega \backslash\left\{x_{1}, \ldots, x_{N}\right\}\right) \text { as } \varepsilon \rightarrow 0
$$

where

$$
G(x)=\sum_{i=1}^{N} \lambda_{i} \mathcal{G}_{h}\left(x_{i}, x\right)
$$

with $\mathcal{G}_{h}$ the Green function of the limit operator $\Delta+h$ with Dirichlet boundary conditions on $\Omega$. From (2.12), we know that $\lambda_{i}>0$ for $1 \leq i \leq N$. This can be rewritten as

$$
G(x)=\frac{\lambda_{i}}{\omega_{2}\left|x-x_{i}\right|}+G_{i}(x)
$$

where $G_{i}$ is a continuous function on $\Omega \backslash\left\{x_{1}, \ldots, x_{i-1}, x_{i+1}, \ldots, x_{N}\right\}$. Thanks to Lem$\mathrm{ma} 4.3$.

$$
G_{i}(x)=G_{i}\left(x_{i}\right)+\frac{h\left(x_{i}\right)}{2 \omega_{2}}\left|x-x_{i}\right|+\gamma_{i}(x)
$$


where $\gamma_{i} \in C^{1}(\Omega)$ and $\gamma_{i}(0)=0$. We claim that

$$
G_{i}\left(x_{i}\right)=0 \quad \text { for all } 1 \leq i \leq N .
$$

In order to prove this, we apply the Pohožaev identity (4.4) to $u_{\varepsilon}$ on the ball $B\left(x_{i, \varepsilon}, \delta\right)$ for some $\delta>0$ small enough. This gives

$$
\begin{aligned}
\frac{1}{2} \int_{B\left(x_{i, \varepsilon}, \delta\right)}\left(h_{\varepsilon} u_{\varepsilon}^{2}\right. & \left.+h_{\varepsilon}\left\langle x-x_{i, \varepsilon}, \nabla u_{\varepsilon}^{2}\right\rangle\right) d x \\
= & \int_{\partial B\left(x_{i, \varepsilon}, \delta\right)}\left(\delta\left(\partial_{\nu} u_{\varepsilon}\right)^{2}-\delta \frac{\left|\nabla u_{\varepsilon}\right|^{2}}{2}+\frac{1}{2} u_{\varepsilon} \partial_{\nu} u_{\varepsilon}+\frac{\delta}{6} u_{\varepsilon}^{6}\right) d \sigma .
\end{aligned}
$$

Thanks to the fact that $h_{\varepsilon}$ is bounded in $L^{p}\left(\mathbb{R}^{3}\right)$ for some $p>3$ and Proposition 1.1 , we get the uniform estimate

$$
u_{\varepsilon}\left(x_{i, \varepsilon}\right)^{2}\left|\frac{1}{2} \int_{B\left(x_{i, \varepsilon}, \delta\right)}\left(h_{\varepsilon} u_{\varepsilon}^{2}+h_{\varepsilon}\left\langle x-x_{i, \varepsilon}, \nabla u_{\varepsilon}^{2}\right\rangle\right) d x\right| \leq e(\delta)
$$

where $e \in C^{0}(\mathbb{R})$ with $e(0)=0$. Using 2.13 , we get

$$
\begin{aligned}
& \int_{\partial B\left(x_{i, \varepsilon}, \delta\right)}\left(\delta\left(\partial_{\nu} u_{\varepsilon}\right)^{2}-\delta \frac{\left|\nabla u_{\varepsilon}\right|^{2}}{2}+\frac{1}{2} u_{\varepsilon} \partial_{\nu} u_{\varepsilon}\right) d \sigma+\int_{\partial B\left(x_{i, \varepsilon}, \delta\right)} \frac{\delta}{6} u_{\varepsilon}^{6} d \sigma \\
& =u_{\varepsilon}\left(x_{i, \varepsilon}\right)^{-2} \int_{\partial B\left(x_{i}, \delta\right)}\left(\delta\left(\partial_{\nu} G\right)^{2}-\delta \frac{|\nabla G|^{2}}{2}+\frac{1}{2} G \partial_{\nu} G\right) d \sigma+o\left(u_{\varepsilon}\left(x_{i, \varepsilon}\right)^{-2}\right)
\end{aligned}
$$

Using 2.14, we easily get

$$
\int_{\partial B\left(x_{i}, \delta\right)}\left(\delta\left(\partial_{\nu} G\right)^{2}-\delta \frac{|\nabla G|^{2}}{2}+\frac{1}{2} G \partial_{\nu} G\right) d \sigma=-\frac{1}{2} \lambda_{i} G_{i}\left(x_{i}\right)+o(1) \quad \text { as } \delta \rightarrow 0 .
$$

Collecting the above information proves 2.15).

We are now going to prove that $\nabla \gamma_{i}\left(x_{i}\right)=0$ where $\gamma_{i}$ is as in 2.14). This will contradict Lemma 4.4 of Appendix 4.4 and complete the proof of the theorem. For that purpose, we apply the Pohožaev identity [4.7] to $u_{\varepsilon}$ on the ball $B\left(x_{i, \varepsilon}, \delta\right)$ for some $\delta>0$ small enough. We obtain

$$
\begin{aligned}
& u_{\varepsilon}\left(x_{i, \varepsilon}\right)^{2} \int_{\partial B\left(x_{i, \varepsilon}, \delta\right)}\left(\frac{\left|\nabla u_{\varepsilon}\right|}{2} v-\nabla u_{\varepsilon} \partial_{\nu} u_{\varepsilon}\right) d \sigma \\
& \quad=u_{\varepsilon}\left(x_{i, \varepsilon}\right)^{2} \int_{B\left(x_{i, \varepsilon}, \delta\right)} h_{\varepsilon} \frac{\nabla u_{\varepsilon}^{2}}{2} d x-u_{\varepsilon}\left(x_{i, \varepsilon}\right)^{2} \int_{\partial B\left(x_{i, \varepsilon}, \delta\right)} \nabla u_{\varepsilon}^{6} d \sigma .
\end{aligned}
$$

It is clear that we can pass to the limit on the left-hand side. Moreover, by 2.15 and 2.14,

$$
\int_{\partial B\left(x_{i}, \delta\right)}\left(\frac{|\nabla G|}{2} \nu-\nabla G \partial_{\nu} G\right) d \sigma \rightarrow \nabla \gamma_{i}\left(x_{i}\right) \quad \text { as } \delta \rightarrow 0 .
$$


Now we look at the right-hand side of 2.17. It is clear that

$$
u_{\varepsilon}\left(x_{i, \varepsilon}\right)^{2} \int_{\partial B\left(x_{i, \varepsilon}, \delta\right)} \nabla u_{\varepsilon}^{6} d \sigma \rightarrow 0 \quad \text { as } \varepsilon \rightarrow 0 .
$$

Then

$$
\int_{B\left(x_{i, \varepsilon}, \delta\right)} h_{\varepsilon} \frac{\nabla u_{\varepsilon}^{2}}{2} d x=\int_{B\left(x_{i, \varepsilon}, \delta\right)}\left(h_{\varepsilon}-h_{\varepsilon}\left(x_{i, \varepsilon}\right)\right) \frac{\nabla u_{\varepsilon}^{2}}{2} d x+h_{\varepsilon}\left(x_{i, \varepsilon}\right) \int_{B\left(x_{i, \varepsilon}, \delta\right)} \frac{\nabla u_{\varepsilon}^{2}}{2} d x .
$$

Assuming that the convergence of $h_{\varepsilon}$ to $h$ holds in $C^{0, \eta}$, it is clear that the first term of the right-hand side goes to 0 as $\varepsilon \rightarrow 0$. Integrating the second term by parts, we get

$$
h_{\varepsilon}\left(x_{i, \varepsilon}\right) \int_{B\left(x_{i, \varepsilon}, \delta\right)} \frac{\nabla u_{\varepsilon}^{2}}{2} d x=h_{\varepsilon}\left(x_{i, \varepsilon}\right) \int_{\partial B\left(x_{i, \varepsilon}, \delta\right)} \frac{u_{\varepsilon}^{2}}{2} v d \sigma \rightarrow h\left(x_{i}\right) \int_{\partial B\left(x_{i}, \delta\right)} \frac{G^{2}}{2} v d \sigma
$$

as $\varepsilon \rightarrow 0$. It is easily checked that the above goes to 0 as $\delta \rightarrow 0$.

Finally, collecting the above information, and passing consecutively to the limit as $\varepsilon \rightarrow 0$ and $\delta \rightarrow 0$ in 2.17, we conclude that $\nabla \gamma_{i}\left(x_{i}\right)=0$ for all $i$, which completes the proof of Theorem 1 thanks to Lemma 4.4 .

Let us now give a precise statement of what we meant by stability of the Pohožaev obstruction in the radial situation in the introduction. We will prove the following:

Theorem 3. Let $B$ be the unit ball of $\mathbb{R}^{3}$. Let $h_{0}$ be a $C^{1}$-radial function which satisfies (0.2). Then for any $p>3$, there exists $\delta>0$ (depending on $h_{0}$ and $p$ ) such that if $h \in C^{0, \eta}(B)$ for some $\eta>0$ with $\left\|h-h_{0}\right\|_{L^{p}(B)} \leq \delta$, then there exists no positive radial solution of equation (0.1) in the unit ball.

Proof. We proceed as in the proof of Theorem 1 . Note that, since $u_{\varepsilon}$ is radial, there can be only one concentration point, namely 0 . Thanks to Claim 2.1, the result of Section 1 and standard elliptic theory,

$$
u_{\varepsilon}(0) u_{\varepsilon}(x) \rightarrow \omega_{2} \mathcal{G}_{h}(x, 0) \quad \text { in } C_{\mathrm{loc}}^{1}(\Omega \backslash\{0\}) \text { as } \varepsilon \rightarrow 0
$$

where $\mathcal{G}_{h}$ is the Green function of the limit operator $\Delta+h$. We have

$$
\mathcal{G}_{h}(x, 0)=\frac{1}{\omega_{2}|x|}+g(x)
$$

where $g$ is a continuous function on $\Omega$ which satisfies

$$
\Delta g+h g=-\frac{h}{\omega_{2}|x|} \quad \text { in } \Omega \text { and } g=-\omega_{2} \quad \text { on } \partial \Omega .
$$

By the maximum principle, we see that $g$ is negative so that $g(0)<0$. Now we can proceed as in the proof of 2.15 to get a contradiction. Note that the proof of 2.15$)$ did not require the $C^{0, \eta}$ convergence of $h_{\varepsilon}$. In the above proof, this $C^{0, \eta}$ convergence was used only in the proof of Claim 2.2 (which is given for free in the radial situation) and in the last part of the proof to deal with the case of several concentration points (which cannot happen in the radial situation).

In the next section we shall prove that the above theorem is sharp in the radial situation. 


\section{Construction of blowing-up examples and instability of the Pohožaev obstruction}

In this section, we prove Theorem 2 In fact, we will first prove the corresponding result in the radial situation (thus showing that our Theorem 3 is sharp) since it contains the main ideas, and the computations are a little less involved.

We first need some results on Green's functions of coercive operators $\Delta+h$ with Dirichlet boundary conditions on domains in $\mathbb{R}^{3}$. We let $\Omega$ be a smooth domain in $\mathbb{R}^{3}$ and $h \in C^{1}(\Omega)$ be such that the operator $\Delta+h$, with Dirichlet boundary conditions, is coercive. Then there exists a unique function $\mathcal{G}: \Omega \times \Omega \backslash\{(x, x): x \in \Omega\} \rightarrow \mathbb{R}$, symmetric, positive, such that

$$
\Delta_{y} \mathcal{G}(x, y)+h(y) \mathcal{G}(x, y)=\omega_{2} \delta_{x}
$$

in $\Omega$ and $\mathcal{G}(x, y)=0$ for $y \in \partial \Omega$ for all $x \in \Omega$. It is easily checked that $\mathcal{G}(x, y)$ has the following expansion in the neighbourhood of the diagonal:

$$
\mathcal{G}(x, y)=\frac{1}{|x-y|}+\frac{1}{2} h(x)|x-y|+\gamma_{x}(y)
$$

where $\gamma_{x} \in C^{1}(\Omega)$ satisfies

$$
\Delta_{y} \gamma_{x}(y)+h(y) \gamma_{x}(y)=\frac{h(x)-h(y)}{|x-y|}-\frac{1}{2} h(x) h(y)|x-y|
$$

in $\Omega$ with

$$
\gamma_{x}(y)=-\frac{1}{|x-y|}-\frac{1}{2} h(x)|x-y| \quad \text { for all } y \in \partial \Omega .
$$

\subsection{The radial case}

We start by proving that the Pohožaev identity is not $L_{r}^{3}$-stable in the unit ball of $\mathbb{R}^{3}$. More precisely, we prove the following result:

Theorem 4. Let $h \in C^{1}(B)$ be a non-negative radial function on the unit ball $B$ of $\mathbb{R}^{3}$. For any $\varepsilon>0$, there exists a radial function $\tilde{h} \in C^{0, \eta}(B)$ with $\|\tilde{h}-h\|_{L^{3}(B)} \leq \varepsilon$ such that the equation

$$
\Delta \tilde{u}+\tilde{h} \tilde{u}=\tilde{u}^{5} \quad \text { in } B, \quad \tilde{u}=0 \quad \text { on } \partial B,
$$

admits a positive radial solution.

Note that a function $h$ which satisfies $(0.2)$ is necessarily non-negative. To prove the theorem, we let $h \in C^{1}(B)$ be a non-negative radial function. We let $\mathcal{G}$ be the Green function of $\Delta+h$ and let $G(x)=\mathcal{G}(0, x)$. We set

$$
u_{\varepsilon}(x)=U_{\varepsilon}(x)+\eta_{\varepsilon}(x) V_{\varepsilon}(x)
$$


where

$$
\begin{aligned}
& U_{\varepsilon}(x)=\varepsilon^{1 / 2}\left(\varepsilon^{2}+G(x)^{-2}\right)^{-1 / 2}, \\
& V_{\varepsilon}(x)=-\gamma_{0}(0) \varepsilon^{1 / 2} G(x)^{-3}\left(\varepsilon^{2}+G(x)^{-2}\right)^{-3 / 2}, \\
& \eta_{\varepsilon}(x)=\eta(x) \frac{\ln \left(\varepsilon^{2}+|x|^{2}\right)}{\ln \varepsilon^{2}} x
\end{aligned}
$$

where $\eta$ is a smooth positive function such that $\eta=1$ on the ball of radius $1 / 4$ and $\eta=0$ outside of the ball of radius $1 / 2$. Here, $\gamma_{0}$ comes from the asymptotic expansion (3.1). It is easily checked that $u_{\varepsilon}$ is a $C^{2, \eta}$ positive function in $B$ and $u_{\varepsilon}=0$ on $\partial B$. Moreover,

$$
\eta_{\varepsilon} V_{\varepsilon} / U_{\varepsilon} \rightarrow 0 \quad \text { in } L^{\infty}(B) \text { as } \varepsilon \rightarrow 0
$$

We claim that

$$
\frac{3 u_{\varepsilon}^{5}-\Delta u_{\varepsilon}}{u_{\varepsilon}} \rightarrow h \quad \text { in } L^{3}(B) \text { as } \varepsilon \rightarrow 0,
$$

which clearly implies the theorem. Straightforward computations give

$$
\Delta U_{\varepsilon}+h U_{\varepsilon}=3 U_{\varepsilon}^{5}\left|\nabla G^{-1}\right|^{2}+h(x) \varepsilon U_{\varepsilon}^{3}
$$

and

$$
\begin{aligned}
\Delta V_{\varepsilon}+h V_{\varepsilon}= & 15 U_{\varepsilon}^{4} V_{\varepsilon}+12 \gamma_{0}(0) \varepsilon^{5 / 2} G^{4}\left(1+\varepsilon^{2} G^{2}\right)^{-5 / 2} \\
& -\varepsilon^{1 / 2} \gamma_{0}(0) h\left(1+\varepsilon^{2} G^{2}\right)^{-5 / 2}\left(1+4 \varepsilon^{2} G^{2}\right) \\
& -3 \gamma_{0}(0) \varepsilon^{5 / 2} G^{4}\left(1+\varepsilon^{2} G^{2}\right)^{-7 / 2}\left(1-4 \varepsilon^{2} G^{2}\right)\left(\left|\nabla G^{-1}\right|^{2}-1\right) .
\end{aligned}
$$

It is easily checked that this implies that

$$
\Delta u_{\varepsilon}+h u_{\varepsilon}-3 u_{\varepsilon}^{5}=o\left(u_{\varepsilon}\right)
$$

in $B_{0}(1) \backslash B_{0}(1 / 2)$. Using the expansion of $G$ and its consequence

$$
\left|\nabla G^{-1}\right|^{2}=1-4 \gamma_{0}(0) G^{-1}+O\left(G^{-2}\right),
$$

we can then write, thanks to 3.4,

$$
\begin{aligned}
\frac{\Delta u_{\varepsilon}+h u_{\varepsilon}-3 u_{\varepsilon}^{5}}{u_{\varepsilon}}= & O\left(|x|^{2} U_{\varepsilon}^{4}\right)+O\left(\varepsilon U_{\varepsilon}^{2}\right)+O\left(|x| U_{\varepsilon}^{4}\left|1-\eta_{\varepsilon}\right|\right) \\
& +O\left(U_{\varepsilon}^{-1}\left|\nabla V_{\varepsilon}\right|\left|\nabla \eta_{\varepsilon}\right|\right)+O\left(U_{\varepsilon}^{-1}\left|V_{\varepsilon}\right|\left|\Delta \eta_{\varepsilon}\right|\right)
\end{aligned}
$$

in $B_{0}(1 / 2)$. It is easily checked that

$$
|x|^{2} U_{\varepsilon}^{4} \rightarrow 0 \quad \text { and } \quad \varepsilon U_{\varepsilon}^{2} \rightarrow 0 \quad \text { in } L^{p}(B) \text { as } \varepsilon \rightarrow 0
$$


for all $1 \leq p<+\infty$. Now,

$$
\begin{aligned}
\int_{B}|x|^{3} U_{\varepsilon}^{12}\left|1-\eta_{\varepsilon}\right|^{3} d x & =O\left(\varepsilon^{6} \int_{0}^{1} r^{5}\left(\varepsilon^{2}+r^{2}\right)^{-6}\left|1-\frac{\ln \left(\varepsilon^{2}+r^{2}\right)}{\ln \varepsilon^{2}}\right|^{3} d r\right) \\
& =O\left(\int_{0}^{\varepsilon^{-1}} r^{5}\left(1+r^{2}\right)^{-6}\left|\frac{\ln \left(1+r^{2}\right)}{\ln \varepsilon^{2}}\right|^{3} d r\right)=O\left(\left|\ln \varepsilon^{2}\right|^{-3}\right) \\
& =O(1)
\end{aligned}
$$

thanks to the dominated convergence theorem. Moreover,

$$
\begin{aligned}
\int_{B} U_{\varepsilon}^{-3}\left|\nabla V_{\varepsilon}\right|^{3}\left|\nabla \eta_{\varepsilon}\right|^{3} d x= & O\left(\left|\ln \varepsilon^{2}\right|^{-3} \int_{0}^{1} r^{11}\left(\varepsilon^{2}+r^{2}\right)^{-6} d r\right) \\
& +O\left(\int_{1 / 4}^{1 / 2}\left|\frac{\ln \left(\varepsilon^{2}+r^{2}\right)}{\ln \varepsilon^{2}}\right|^{3} d r\right) \\
= & O\left(\left|\ln \varepsilon^{2}\right|^{-3}\right)=o(1)
\end{aligned}
$$

and

$$
\begin{aligned}
\int_{B} U_{\varepsilon}^{-3}\left|V_{\varepsilon}\right|^{3}\left|\Delta \eta_{\varepsilon}\right|^{3} d x & =O\left(\left|\ln \varepsilon^{2}\right|^{-3} \int_{0}^{1} r^{5}\left(\varepsilon^{2}+r^{2}\right)^{-1} d r\right)+O\left(\left|\ln \varepsilon^{2}\right|^{-3}\right) \\
& =O\left(\left|\ln \varepsilon^{2}\right|^{-3}\right)=o(1) .
\end{aligned}
$$

Coming back to (3.9) with these last estimates, we get 3.5). This ends the proof of Theorem 4

\subsection{The general case}

Here we prove that the Pohožaev identity is never $L^{\infty}$-stable. In fact we will even prove a stronger result:

Theorem 5. Let $\Omega$ be a smooth domain of $\mathbb{R}^{3}$ and let $h \in C^{1}(\Omega)$ be such that the operator $\Delta+h$ is coercive. For any $\varepsilon>0$, there exists $\tilde{h} \in C^{0, \eta}(\Omega)$ with $\|\tilde{h}-h\|_{\infty} \leq \varepsilon$ such that the equation

$$
\left\{\begin{array}{l}
\Delta \tilde{u}+\tilde{h} \tilde{u}=\tilde{u}^{5} \quad \text { in } \Omega, \\
\tilde{u}=0 \quad \text { on } \partial \Omega, \quad \tilde{u}>0 \quad \text { in } \Omega,
\end{array}\right.
$$

admits a solution.

It is clear that this result implies Theorem 2 It is sufficient to remember that a function $h$ which satisfies 0.2 is necessarily non-negative and that a non-negative $h$ leads to a coercive operator $\Delta+h$. The rest of this subsection is devoted to the proof of this theorem.

We will construct a sequence of functions $u_{\varepsilon} \in C^{\infty}(\Omega)$, positive in $\Omega$, null on the boundary of $\Omega$, such that

$$
\frac{\Delta u_{\varepsilon}-3 u_{\varepsilon}^{5}}{u_{\varepsilon}} \rightarrow h \quad \text { in } L^{\infty}(\Omega) \text { as } \varepsilon \rightarrow 0
$$

This will clearly prove the theorem. 
We let $\mathcal{G}$ be the Green function of the operator $\Delta+h$ in $\Omega$ with Dirichlet boundary conditions. Note first that $\gamma_{x}(x) \rightarrow-\infty$ as $x$ approaches $\partial \Omega$. In particular, there exists a point $x_{1} \in \Omega$ such that $\gamma_{x_{1}}\left(x_{1}\right)<0$. For $x \in \Omega \backslash\left\{x_{1}\right\}$, we set

$$
\lambda(x)=\left(-\frac{\gamma_{x_{1}}\left(x_{1}\right)}{\mathcal{G}\left(x_{1}, x\right)}\right)^{2}, \quad F(x)=\mathcal{G}\left(x_{1}, x\right)^{2}-\gamma_{x_{1}}\left(x_{1}\right) \gamma_{x}(x) .
$$

Since $F(x) \rightarrow+\infty$ as $x \rightarrow x_{1}$ and $F(x) \rightarrow-\infty$ as $x$ approaches $\partial \Omega$, and since $F$ is continuous, there exists $x_{2}$ such that $F\left(x_{2}\right)=0$. We then let $\lambda=\lambda\left(x_{2}\right)$ and we have

$$
\sqrt{\lambda} G_{2}\left(x_{1}\right)+\gamma_{1}\left(x_{1}\right)=G_{1}\left(x_{2}\right)+\sqrt{\lambda} \gamma_{2}\left(x_{2}\right)=0
$$

where

$$
G_{1}(x)=\mathcal{G}\left(x_{1}, x\right), \quad G_{2}(x)=\mathcal{G}\left(x_{2}, x\right), \quad \gamma_{1}(x)=\gamma_{x_{1}}(x), \quad \gamma_{2}(x)=\gamma_{x_{2}}(x) .
$$

We let $\delta>0$ be such that $\delta \leq 10 d\left(x_{1}, \partial \Omega\right)$ and $\delta \leq 10 d\left(x_{2}, \partial \Omega\right)$. We fix $\eta \in C^{\infty}(\mathbb{R})$ such that $\eta(r)=1$ for $|r| \leq \delta$ and $\eta(r)=0$ for $|r| \geq 2 \delta$. We set

$$
\begin{aligned}
& u_{\varepsilon}=\varepsilon^{-1 / 2} U\left(\varepsilon G_{1}\right)+(\lambda \varepsilon)^{-1 / 2} U\left(\lambda \varepsilon G_{2}\right) \\
& +\eta\left(\left|x-x_{1}\right|\right) \gamma_{1}\left(x_{1}\right) \varepsilon^{1 / 2} V\left(\varepsilon G_{1}\right)+\eta\left(\left|x-x_{2}\right|\right) \gamma_{2}\left(x_{2}\right)(\lambda \varepsilon)^{1 / 2} V\left(\lambda \varepsilon G_{2}\right) \\
& -\eta\left(\left|x-x_{1}\right|\right) \psi_{\varepsilon}\left(\varepsilon G_{1}\right) \varepsilon^{1 / 2}\left(x-x_{1}\right)^{i}\left(\left(1+\varepsilon^{2} G_{1}^{2}\right)^{-3 / 2} \partial_{i} \gamma_{1}\left(x_{1}\right)+\lambda^{1 / 2} \partial_{i} G_{2}\left(x_{1}\right)\right) \\
& -\eta\left(\left|x-x_{2}\right|\right) \psi_{\varepsilon}\left(\lambda \varepsilon G_{2}\right)(\lambda \varepsilon)^{1 / 2}\left(x-x_{2}\right)^{i}\left(\left(1+\lambda^{2} \varepsilon^{2} G_{2}^{2}\right)^{-3 / 2} \partial_{i} \gamma_{2}\left(x_{2}\right)+\lambda^{-1 / 2} \partial_{i} G_{1}\left(x_{2}\right)\right) \\
& +\eta\left(\left|x-x_{1}\right|\right) \varepsilon^{3 / 2} \psi_{\varepsilon}\left(\varepsilon G_{1}\right)\left(h\left(x_{1}\right) W\left(\varepsilon G_{1}\right)-\frac{3}{2} \gamma_{1}\left(x_{1}\right)^{2} U\left(\varepsilon G_{1}\right)^{5}\right) \\
& +\eta\left(\left|x-x_{2}\right|\right)(\lambda \varepsilon)^{3 / 2} \psi_{\varepsilon}\left(\lambda \varepsilon G_{2}\right)\left(h\left(x_{2}\right) W\left(\lambda \varepsilon G_{2}\right)-\frac{3}{2} \gamma_{2}\left(x_{2}\right)^{2} U\left(\lambda \varepsilon G_{2}\right)^{5}\right)
\end{aligned}
$$

where we adopt Einstein's summation convention and $U, V, W$ and $\psi_{\varepsilon}$ are given by

$$
\begin{aligned}
& U(r)=r\left(1+r^{2}\right)^{-1 / 2}, \quad V(r)=1-\left(1+r^{2}\right)^{-3 / 2}, \quad \psi_{\varepsilon}(r)=1+\frac{\ln \left(1+r^{-2}\right)}{\ln \varepsilon^{2}}, \\
& W(r)=-\frac{13}{4} U+8\left(2 U^{3}-U\right) \ln U-2\left(U^{-1}-8 U+8 U^{3}\right) r \arctan \left(\frac{1}{r}\right) .
\end{aligned}
$$

It is easily checked that $u_{\varepsilon}$ is $C^{2, \eta}$ in $\Omega$ and $u_{\varepsilon}=0$ on $\partial \Omega$. We now claim that 3.11 holds for this specific $u_{\varepsilon}$ and that $u_{\varepsilon}$ is positive in $\Omega$. We shall prove this claim in three steps. First, we can prove it rather easily in $\Omega \backslash\left(B_{x_{1}}(2 \delta) \cup B_{x_{2}}(2 \delta)\right)$ because, in this region, $u_{\varepsilon}$ is simply

$$
u_{\varepsilon}=\varepsilon^{-1 / 2} U\left(\varepsilon G_{1}\right)+(\lambda \varepsilon)^{-1 / 2} U\left(\lambda \varepsilon G_{2}\right) \text {. }
$$

Now, noticing that $U^{\prime}=r^{-3} U^{3}$ and that $U^{\prime \prime}=-3 r^{-4} U^{5}$, simple computations lead to

$$
\begin{aligned}
\Delta\left(\varepsilon^{-1 / 2} U\left(\varepsilon G_{1}\right)+(\lambda \varepsilon)^{-1 / 2} U\left(\lambda \varepsilon G_{2}\right)\right)+h\left(\varepsilon^{-1 / 2} U\left(\varepsilon G_{1}\right)+(\lambda \varepsilon)^{-1 / 2} U\left(\lambda \varepsilon G_{2}\right)\right) \\
=3\left(\varepsilon^{-1 / 2} U\left(\varepsilon G_{1}\right)\right)^{5}\left|\nabla G_{1}^{-1}\right|^{2}+3\left((\lambda \varepsilon)^{-1 / 2} U\left(\lambda \varepsilon G_{2}\right)\right)^{5}\left|\nabla G_{2}^{-1}\right|^{2} \\
\quad+h \varepsilon^{-1 / 2} U\left(\varepsilon G_{1}\right)\left(1-\left(1+\varepsilon^{2} G_{1}^{2}\right)^{-1}\right) \\
+h(\lambda \varepsilon)^{-1 / 2} U\left(\lambda \varepsilon G_{2}\right)\left(1-\left(1+\lambda^{2} \varepsilon^{2} G_{2}^{2}\right)^{-1}\right)
\end{aligned}
$$


in $\Omega$. In the region we are interested in, this clearly leads to

$$
\Delta u_{\varepsilon}+h u_{\varepsilon}-3 u_{\varepsilon}^{5}=o\left(u_{\varepsilon}\right)
$$

which proves that 3.11 holds in this region while $u_{\varepsilon}$ is clearly positive there.

We will now prove that 3.11 holds in $B_{x_{1}}(2 \delta)$ and that $u_{\varepsilon}$ is positive in this ball. By symmetry, it is clear that the proof of the fact that 3.11 holds in $B_{x_{2}}(2 \delta)$ is exactly the same ${ }^{3}$ In order to simplify the notations, we will assume that $x_{1}=0$, which we can always do by translating $\Omega$. We will denote $G_{1}$ by $G$ and $\gamma_{1}$ by $\gamma$. We also set

$$
\begin{aligned}
& U_{\varepsilon}=\varepsilon^{-1 / 2} U(\varepsilon G), \quad V_{\varepsilon}=\varepsilon^{1 / 2} V(\varepsilon G), \quad W_{\varepsilon}=\varepsilon^{3 / 2} W(\varepsilon G), \\
& \varphi_{\varepsilon}=\psi_{\varepsilon}(\varepsilon G), \quad Y_{\varepsilon}=-\frac{3}{2} \varepsilon^{3 / 2} U(\varepsilon G)^{5}, \quad \tilde{U}_{\varepsilon}=(\lambda \varepsilon)^{-1 / 2} U\left(\lambda \varepsilon G_{2}\right), \\
& Z_{\varepsilon}=-\varepsilon^{1 / 2} x^{i}\left(\left(1+\varepsilon^{2} G^{2}\right)^{-3 / 2} \partial_{i} \gamma(0)+\lambda^{1 / 2} \partial_{i} G_{2}(0)\right) .
\end{aligned}
$$

With these notations, we have, in $B_{0}(2 \delta)$,

$$
u_{\varepsilon}=U_{\varepsilon}+\tilde{U}_{\varepsilon}+\eta(|x|) \gamma(0) V_{\varepsilon}+\eta(|x|) \varphi_{\varepsilon}\left(h(0) W_{\varepsilon}+\gamma(0)^{2} Y_{\varepsilon}+Z_{\varepsilon}\right)
$$

By (3.1),

$$
\begin{aligned}
\left|\nabla G^{-1}\right|^{2}= & 1-4 \gamma(0) G^{-1}+3\left(2 \gamma(0)^{2}-h(0)\right) G^{-2} \\
& -6 G^{-1} x^{i} \partial_{i} \gamma(0)+o\left(G^{-2}\right)
\end{aligned}
$$

and also

$$
G^{-2}=\varepsilon U_{\varepsilon}^{-2}-\varepsilon^{2}
$$

By 3.12,

$$
\begin{aligned}
& \tilde{U}_{\varepsilon}=-\varepsilon^{1 / 2} \gamma(0)+(\lambda \varepsilon)^{1 / 2} x^{i} \partial_{i} G_{2}(0)+O\left(\varepsilon^{3 / 2} U_{\varepsilon}^{-2}\right), \\
& V_{\varepsilon}=O\left(\varepsilon^{3 / 2} U_{\varepsilon}^{2}\right), \quad W_{\varepsilon}=O\left(\varepsilon^{3 / 2}\right), \quad Y_{\varepsilon}=O\left(\varepsilon^{3 / 2}\right), \quad Z_{\varepsilon}=O\left(\varepsilon U_{\varepsilon}^{-1}\right) .
\end{aligned}
$$

From 3.18 and to 3.21, it is easily checked that $u_{\varepsilon}$ is positive in $B_{0}(2 \delta)$. Lengthy but straightforward computations then lead to

$$
\begin{aligned}
& \left|\nabla \varphi_{\varepsilon}\right|=O\left(\frac{1}{\varepsilon^{1 / 2} \ln (1 / \varepsilon)} U_{\varepsilon}\right), \quad\left|\nabla V_{\varepsilon}\right|=O\left(\varepsilon U_{\varepsilon}^{3}\right), \quad\left|\nabla W_{\varepsilon}\right|=O\left(\varepsilon U_{\varepsilon}\right), \\
& \left|\nabla Y_{\varepsilon}\right|=O\left(\varepsilon U_{\varepsilon}\right), \quad\left|\nabla Z_{\varepsilon}\right|=O\left(\varepsilon^{1 / 2}\right)
\end{aligned}
$$

\footnotetext{
3 The symmetry is precisely the following: if we see $u_{\varepsilon}$ as a function $u_{\varepsilon}\left(x_{1}, x_{2}, \varepsilon, \lambda\right)$, then $u_{\varepsilon}\left(x_{2}, x_{1}, \lambda \varepsilon, \lambda^{-1}\right)=u_{\varepsilon}\left(x_{1}, x_{2}, \varepsilon, \lambda\right)$.
} 
in $B_{0}(2 \delta)$ and to

$$
\begin{aligned}
\Delta U_{\varepsilon}+h U_{\varepsilon}= & 3 U_{\varepsilon}^{5}-12 \gamma(0) G^{-1} U_{\varepsilon}^{5}-18 G^{-1} x^{i} \partial_{i} \gamma(0) U_{\varepsilon}^{5} \\
& +18 \gamma(0)^{2} G^{-2} U_{\varepsilon}^{5}+h(0)\left(\varepsilon^{2}-8 G^{-2}\right) U_{\varepsilon}^{5}+o\left(U_{\varepsilon}\right), \\
\Delta \tilde{U}_{\varepsilon}+h \tilde{U}_{\varepsilon}= & 3 \tilde{U}_{\varepsilon}^{5}\left|\nabla G_{2}^{-1}\right|^{2}+\lambda \varepsilon h \tilde{U}_{\varepsilon}^{3}=O\left(\varepsilon^{5 / 2}\right) \\
\Delta V_{\varepsilon}+h V_{\varepsilon}= & 15 U_{\varepsilon}^{4} V_{\varepsilon}-15 \varepsilon^{1 / 2} U_{\varepsilon}^{4}+12 G^{-1} U_{\varepsilon}^{5} \\
& +12 \gamma(0)\left(5 \varepsilon^{-1} G^{-4} U_{\varepsilon}^{7}-4 G^{-2} U_{\varepsilon}^{5}\right)+o\left(U_{\varepsilon}\right), \\
\Delta W_{\varepsilon}+h W_{\varepsilon}= & 15 U_{\varepsilon}^{4} W_{\varepsilon}+8 \varepsilon U_{\varepsilon}^{3}-9 \varepsilon^{2} U_{\varepsilon}^{5}+o\left(U_{\varepsilon}\right), \\
\Delta Y_{\varepsilon}+h Y_{\varepsilon}= & 15 U_{\varepsilon}^{4} Y_{\varepsilon}+30 \varepsilon^{3} U_{\varepsilon}^{7}-30 \varepsilon^{4} U_{\varepsilon}^{9}+o\left(U_{\varepsilon}\right), \\
\Delta \varphi_{\varepsilon}= & O\left(\frac{1}{\varepsilon \ln (1 / \varepsilon)} U_{\varepsilon}^{2}\right) \\
\Delta Z_{\varepsilon}+h Z_{\varepsilon}= & 15 U_{\varepsilon}^{4} Z_{\varepsilon}+18 U_{\varepsilon}^{5} G^{-1} \partial_{i} \gamma(0) x^{i} \\
& +15(\lambda \varepsilon)^{1 / 2} U_{\varepsilon}^{4} x^{i} \partial_{i} G_{2}(0)+O\left(\varepsilon U_{\varepsilon}^{-1}\right)+o\left(U_{\varepsilon}\right)
\end{aligned}
$$

in $B_{0}(2 \delta)$. It follows easily from the above equations that

$$
\frac{\Delta u_{\varepsilon}+h u_{\varepsilon}-3 u_{\varepsilon}^{5}}{u_{\varepsilon}} \rightarrow 0 \quad \text { in } L^{\infty}\left(B_{0}(2 \delta) \backslash B_{0}(\delta)\right) \text { as } \varepsilon \rightarrow 0 .
$$

It remains to prove the result in $B_{0}(\delta)$. Thanks to 3.21 , one can easily check that

$$
\frac{u_{\varepsilon}}{U_{\varepsilon}} \rightarrow 1+\sqrt{\lambda} \frac{G_{2}}{G_{1}} \quad \text { in } L^{\infty}\left(B_{0}(\delta)\right) \text { as } \varepsilon \rightarrow 0
$$

so that

$$
3 u_{\varepsilon}^{5}=3 U_{\varepsilon}^{5}+15 U_{\varepsilon}^{4}\left(u_{\varepsilon}-U_{\varepsilon}\right)+30 U_{\varepsilon}^{3}\left(u_{\varepsilon}-U_{\varepsilon}\right)^{2}+O\left(U_{\varepsilon}^{2}\left|u_{\varepsilon}-U_{\varepsilon}\right|^{3}\right) .
$$

Using again 3.22, we deduce that

$$
\begin{aligned}
3 u_{\varepsilon}^{5}= & 3 U_{\varepsilon}^{5}-15 \varepsilon^{1 / 2} \gamma(0) U_{\varepsilon}^{4}+15(\lambda \varepsilon)^{1 / 2} U_{\varepsilon}^{4} x^{i} \partial_{i} G_{2}(0) \\
& +15 \gamma(0) U_{\varepsilon}^{4} V_{\varepsilon}+15 U_{\varepsilon}^{4} \varphi_{\varepsilon}\left(h(0) W_{\varepsilon}+\gamma(0)^{2} Y_{\varepsilon}+Z_{\varepsilon}\right) \\
& +30 \gamma(0)^{2} U_{\varepsilon}^{3}\left(V_{\varepsilon}-\varepsilon^{1 / 2}\right)^{2}+o\left(U_{\varepsilon}\right)
\end{aligned}
$$

in $B_{0}(\delta)$. Thanks to 3.21 -3.23 , we can also write

$$
\begin{aligned}
\Delta u_{\varepsilon}+h u_{\varepsilon}= & 3 U_{\varepsilon}^{5}+15 \gamma(0) U_{\varepsilon}^{4} V_{\varepsilon}+15(\lambda \varepsilon)^{1 / 2} \varphi_{\varepsilon} U_{\varepsilon}^{4} x^{i} \partial_{i} G_{2}(0) \\
& -15 \gamma(0) \varepsilon^{1 / 2} U_{\varepsilon}^{4}+15 U_{\varepsilon}^{4} \varphi_{\varepsilon}\left(h(0) W_{\varepsilon}+\gamma(0)^{2} Y_{\varepsilon}+Z_{\varepsilon}\right) \\
& +30 \gamma(0)^{2}\left(2 \varepsilon^{-1} G^{-4} U_{\varepsilon}^{7}-G^{-2} U_{\varepsilon}^{5}\right)+h(0)\left(\varepsilon^{2}-8 G^{-2}\right) U_{\varepsilon}^{5} \\
& +h(0) \varphi_{\varepsilon}\left(8 \varepsilon U_{\varepsilon}^{3}-9 \varepsilon^{2} U_{\varepsilon}^{5}\right)+30 \gamma(0)^{2} \varphi_{\varepsilon}\left(\varepsilon^{3} U_{\varepsilon}^{7}-\varepsilon^{4} U_{\varepsilon}^{9}\right) \\
& +18\left(\varphi_{\varepsilon}-1\right) U_{\varepsilon}^{5}|x| \partial_{i} \gamma(0) x^{i}+o\left(U_{\varepsilon}\right) .
\end{aligned}
$$


Combining these last two equations, we get

$$
\begin{aligned}
\Delta u_{\varepsilon}+ & h u_{\varepsilon}-3 u_{\varepsilon}^{5} \\
= & -30 \gamma(0)^{2}\left(U_{\varepsilon}^{3}\left(V_{\varepsilon}-\varepsilon^{1 / 2}\right)^{2}-\varphi_{\varepsilon} \varepsilon^{3} U_{\varepsilon}^{7}+\varphi_{\varepsilon} \varepsilon^{4} U_{\varepsilon}^{9}-2 \varepsilon^{-1} G^{-4} U_{\varepsilon}^{7}+G^{-2} U_{\varepsilon}^{5}\right) \\
& +h(0)\left(\varepsilon^{2} U_{\varepsilon}^{5}-8 G^{-2} U_{\varepsilon}^{5}+8 \varphi_{\varepsilon} \varepsilon U_{\varepsilon}^{3}-9 \varphi_{\varepsilon} \varepsilon^{2} U_{\varepsilon}^{5}\right)+o\left(U_{\varepsilon}\right) \\
& +18\left(\varphi_{\varepsilon}-1\right)|x| x^{i} \partial_{i} \gamma(0) U_{\varepsilon}^{5} .
\end{aligned}
$$

It remains to remark using 3.20 ) that

$$
\begin{aligned}
U_{\varepsilon}^{3}\left(V_{\varepsilon}\right. & \left.-\varepsilon^{1 / 2}\right)^{2}-\varphi_{\varepsilon} \varepsilon^{3} U_{\varepsilon}^{7}+\varphi_{\varepsilon} \varepsilon^{4} U_{\varepsilon}^{9}-2 \varepsilon^{-1} G^{-4} U_{\varepsilon}^{7}+G^{-2} U_{\varepsilon}^{5} \\
& =\varepsilon^{2} G^{-2} U_{\varepsilon}^{9}\left(1-\varphi_{\varepsilon}\right)=-\frac{\varepsilon^{2}}{\ln \varepsilon^{2}} \ln \left(1+\varepsilon^{-2} G^{-2}\right) G^{-2} U_{\varepsilon}^{9} \\
& =-\frac{U_{\varepsilon}}{\ln \varepsilon^{2}} \varepsilon^{6} \ln \left(1+\varepsilon^{-2} G^{-2}\right) G^{-2}\left(\varepsilon^{2}+G^{-2}\right)^{-4}=O\left(\frac{U_{\varepsilon}}{\ln (1 / \varepsilon)}\right)=o\left(U_{\varepsilon}\right),
\end{aligned}
$$

that

$$
\begin{aligned}
& \varepsilon^{2} U_{\varepsilon}^{5}-8 G^{-2} U_{\varepsilon}^{5}+8 \varphi_{\varepsilon} \varepsilon U_{\varepsilon}^{3}-9 \varphi_{\varepsilon} \varepsilon^{2} U_{\varepsilon}^{5}=\left(-9 \varepsilon^{2} U_{\varepsilon}^{5}+8 \varepsilon U_{\varepsilon}^{3}\right)\left(\varphi_{\varepsilon}-1\right) \\
& =\frac{U_{\varepsilon}}{\ln \varepsilon^{2}} \ln \left(1+\varepsilon^{-2} G^{-2}\right)\left(-9\left(1+\varepsilon^{-2} G^{-2}\right)^{-2}+8\left(1+\varepsilon^{2} G^{-2}\right)^{-1}\right) \\
& =O\left(\frac{U_{\varepsilon}}{\ln (1 / \varepsilon)}\right)=o\left(U_{\varepsilon}\right),
\end{aligned}
$$

and that

$$
\begin{aligned}
\left(\varphi_{\varepsilon}-1\right) G^{-1} x^{i} \partial_{i} \gamma(0) U_{\varepsilon}^{5} & =O\left(\frac{U_{\varepsilon}}{\ln \varepsilon^{2}} \ln \left(1+\varepsilon^{-2} G^{-2}\right) \varepsilon^{-2} G^{-2}\left(1+\varepsilon^{-2} G^{-2}\right)^{-2}\right) \\
& =O\left(\frac{U_{\varepsilon}}{\ln (1 / \varepsilon)}\right)=o\left(U_{\varepsilon}\right)
\end{aligned}
$$

to conclude thanks to 3.24 that 3.11 holds in $B_{0}(\delta)$ for this choice of $u_{\varepsilon}$. As already said, this proves that (3.11) holds for $u_{\varepsilon}$ given by (3.14), and this ends the proof of the theorem.

As already said, this result implies Theorem 2

\section{Appendix}

\subsection{A general simple lemma on functions}

We prove a new version of the simple Lemma 1.1 of [8], replacing the compact manifold $M$ by a domain $\Omega$ in $\mathbb{R}^{n}$. 
Lemma 4.1. Let $\Omega$ be a smooth bounded domain in $\mathbb{R}^{n}$. Let $u \in C^{1}(\Omega)$ be a function positive in the interior and null on the boundary. Assume that

$$
\left\{x \in \Omega: \nabla u(x)=0 \text { and } d(x, \partial \Omega) u(x)^{2} \geq 1\right\} \neq \emptyset .
$$

Then there exist $N \in \mathbb{N}^{*}$ and $N$ critical points of $u$, denoted by $\left(x_{1}, \ldots, x_{N}\right)$, such that

$$
\begin{array}{ll}
d\left(x_{i}, \partial \Omega\right) u\left(x_{i}\right)^{2} \geq 1 & \text { for all } i \in[1, N], \\
\left|x_{i}-x_{j}\right| u\left(x_{i}\right)^{2} \geq 1 & \text { for all } i \neq j \in[1, N],
\end{array}
$$

and

$$
\min _{i \in[1, N]}\left|x_{i}-x\right| u(x)^{2} \leq 1
$$

for all critical points $x$ of $u$ such that $d(x, \partial \Omega) u(x)^{2} \geq 1$.

Proof. Let $\mathcal{C}_{u}$ be the set of critical points of $u$. We let

$$
K_{0}=\left\{x \in \mathcal{C}_{u}: d(x, \partial \Omega) u(x)^{2} \geq 1\right\}
$$

and we assume that $K_{0} \neq \emptyset$. We let $x_{1} \in K_{0}$ and $K_{1} \subset K_{0}$ be such that

$$
u\left(x_{1}\right)=\max _{K_{0}} u \quad \text { and } \quad K_{1}=\left\{x \in K_{0}:\left|x_{1}-x\right| u(x)^{2} \geq 1\right\} .
$$

Then we proceed by induction. Assuming we have constructed $K_{0} \supset \cdots \supset K_{p}$ and $x_{1}, \ldots, x_{p}$ such that $x_{i} \in K_{i-1}$ for all $i \in[1, p]$, we let $x_{p+1} \in K_{p}$ and $K_{p+1} \subset K_{p}$ be such that

$$
u\left(x_{p+1}\right)=\max _{K_{p}} u
$$

and

$$
K_{p+1}=\left\{x \in K_{p}:\left|x_{p+1}-x\right| u\left(x_{p+1}\right)^{2} \geq 1 \text { and } \min _{i \in[1, p]}\left|x-x_{i}\right| u(x)^{2} \geq 1\right\} .
$$

We claim that, at some step in the process, $K_{p}=\emptyset$. In order to prove it, we remark that at each step of the construction,

$$
\left|x_{i}-x_{j}\right| u\left(x_{i}\right)^{2} \geq 1 \quad \text { for all } i \neq j \in[1, p],
$$

which will prove the claim, since $\Omega$ is bounded. We prove (4.1) by induction. Let $p \geq 1$. By definition, for all $x \in K_{p}$, we have

$$
\left|x_{i}-x\right| u(x)^{2} \geq 1 \quad \text { for all } i \in[1, p] .
$$

This holds in particular for $x=x_{p+1}$. Then, for all $x \in K_{p}$, we also easily check that

$$
\left|x_{i}-x\right| u\left(x_{i}\right)^{2} \geq 1 \quad \text { for all } i \in[1, p],
$$

which is also true for $x_{p+1}$, and proves 4.1. Let $N \in \mathbb{N}^{*}$ be such that $K_{N}=\emptyset$. We claim that

$$
\min _{i \in[1, N]}\left|x_{i}-x\right| u(x)^{2} \leq 1 \quad \text { for all } x \in K_{0},
$$


which, together with (4.1), will end the proof of the lemma. Let $x \in K_{0}$. Since $K_{N}=\emptyset$, there exists $p$ such that $x \in K_{p-1}$ and $x \notin K_{p}$. Then either

$$
\left|x_{p}-x\right| u\left(x_{p}\right)^{2}<1 \quad \text { or } \min _{i \in[1, p]}\left|x-x_{i}\right| u(x)^{2}<1 .
$$

In the second case, (4.2) is clearly true, while in the first, by the definition of $x_{p}$,

$$
\left|x_{p}-x\right| u(x)^{2} \leq\left|x_{p}-x\right| u\left(x_{p}\right)^{2}<1,
$$

which proves that 4.2 also holds. As already said, this proves the lemma.

\subsection{Green function of $\Delta+h$}

We prove here some basic estimates on Green's functions of operators $\Delta+h$ where $h$ is of low regularity.

Lemma 4.2. Let $\Omega$ be a smooth bounded domain in $\mathbb{R}^{3}$. Let $h \in L^{p}(\Omega)$ for some $p>3$. Then there exists $\delta>0$ such that if

$$
\left\|h_{-}\right\|_{3 / 2}<\delta,
$$

then the operator $\Delta+h$ admits a positive Green function $\mathcal{G}_{h}$ which satisfies the estimates

$$
|| x-y\left|\mathcal{G}_{h}(x, y)-\frac{1}{\omega_{2}}\right| \leq C|x-y|
$$

and

$$
|| x-\left.y\right|^{2}\left|\nabla \mathcal{G}_{h}(x, y)\right|-\frac{1}{\omega_{2}}|\leq C| x-y \mid
$$

for all $x \neq y \in \Omega$, where $C$ is a positive constant depending only on $\Omega,\|h\|_{p}$ and $\delta$.

Proof. We divide the proof into three steps.

Step 1: $\Delta+h$ is coercive if $\left\|h_{-}\right\|_{3 / 2}$ is small enough. Let $u \in H_{0}^{1}(\Omega)$. Then

$$
\int_{\Omega}\left(|\nabla u|^{2}+h u^{2}\right) d x \geq \int_{\Omega}\left(|\nabla u|^{2}-h_{-} u^{2}\right) d x \geq\|\nabla u\|_{2}^{2}-\left\|h_{-}\right\|_{3 / 2}\|u\|_{6}
$$

thanks to Hölder's inequalities. One can then use Sobolev's embeddings and the fact that $\left\|h_{-}\right\|_{3 / 2}$ is small to conclude this first step.

Step 2: Existence and a priori estimate. Let $\mathcal{G}(x, y)$ be the Green function of the Laplacian. Then solving

$$
\begin{aligned}
\Delta_{y} \mathcal{G}_{h}(x, y)+h \mathcal{G}_{h}(x, y) & =\delta_{x} & & \text { in } \Omega, \\
\mathcal{G}_{h}(x, y) & =0 & & \text { on } \partial \Omega,
\end{aligned}
$$

is equivalent to solving

$$
\begin{aligned}
\Delta_{y} \beta(x, y)+h \beta(x, y) & =-h \mathcal{G}(x, y), \\
\beta(x, y) & =0 \quad \text { on } \partial \Omega .
\end{aligned}
$$


Since $h \in L^{p}(\Omega)$ for some $p>3$, there exists $q>3 / 2$ such that $h \mathcal{G}(x, \cdot) \in L^{q}(\Omega)$. The existence of $\beta$ follows from the coercivity of $\Delta+h$ and the Lax-Milgram theorem. Moreover, using again the coercivity of $\Delta+h$ and Sobolev's embeddings, we get

$$
\frac{1}{C}\|\nabla \beta\|_{2}^{2} \leq \int_{\Omega}\left(|\nabla \beta|^{2}+h \beta^{2}\right) d x=\int_{\Omega}-h \mathcal{G} \beta d x \leq\|h \mathcal{G}\|_{3 / 2}\|\beta\|_{3} \leq C\|\nabla \beta\|_{2}
$$

for some $C>1$ depending only on $\|h\|_{p},\left\|h_{-}\right\|_{3 / 2}$ and $\Omega$. This gives an a priori bound on $\|\nabla \beta\|_{2}$.

Step 3: Estimates and positivity. By the previous step, there exists $C>0$ which depends only on $\|h\|_{p}$ and $\left\|h_{-}\right\|_{3 / 2}$, and $q>3 / 2$ such that

$$
\|h(\beta+\mathcal{G}(x, \cdot))\|_{q} \leq C .
$$

Now, by standard elliptic theory (see for instance Theorem 9.13 of [9]), we see that $\beta \in L^{\infty}$ and

$$
\|\beta\|_{\infty} \leq C
$$

where $C$ is a positive constant which depends only on $\|h\|_{p}$ and $\left\|h_{-}\right\|_{3 / 2}$. This proves the first estimate of the lemma. The second follows by standard elliptic theory. Positivity of the Green function is an easy consequence of the coercivity of the operator $\Delta+h$.

\subsection{General Pohožaev identities}

For the sake of completeness, we derive here several forms of the classical Pohožaev identity [11] we used in this paper. Assume that $u$ is a $C^{2}$ solution of

$$
\Delta u=u^{5}-h u \quad \text { in } \Omega \text {. }
$$

Multiplying this equation by $\langle x, \nabla u\rangle$ and integrating by parts, one easily gets

$$
\frac{1}{2} \int_{\Omega}\left(h u^{2}+h\left\langle x, \nabla u^{2}\right\rangle\right) d x=B_{1}+B_{2}
$$

where

$$
\begin{aligned}
& B_{1}=\int_{\partial \Omega}\left(\langle x, \nabla u\rangle \partial_{\nu} u+\frac{1}{2} u \partial_{\nu} u-\langle x, \nu\rangle \frac{|\nabla u|^{2}}{2}\right) d \sigma, \\
& B_{2}=\int_{\partial \Omega}\langle x, v\rangle \frac{u^{6}}{6} d \sigma .
\end{aligned}
$$

Hence, if $u=0$ on $\partial \Omega$, we get

$$
\frac{1}{2} \int_{\Omega} h\left(u^{2}+\left\langle x, \nabla u^{2}\right\rangle\right) d x=\int_{\partial \Omega}\langle x, v\rangle\left(\partial_{\nu} u\right)^{2} d \sigma .
$$

Integrating by parts again, we deduce the Pohožaev identity in its usual form:

$$
\int_{\Omega}\left(h+\frac{\langle x, \nabla h\rangle}{2}\right) u^{2} d x=-\int_{\partial \Omega}\langle x, v\rangle\left(\partial_{\nu} u\right)^{2} d \sigma .
$$


In a similar way, multiplying the equation by $\nabla u$ and integrating by parts, one can derive the following Pohožaev identity:

$$
\int_{\partial \Omega}\left(\frac{|\nabla u|^{2}}{2} v-\partial_{\nu} u \nabla u+\frac{u^{6}}{6} v\right) d \sigma=\int_{\Omega} h \frac{\nabla u^{2}}{2} d x
$$

\subsection{Pohožaev's identity for Green functions}

In this section, we prove a useful Pohožaev identity for a sum of Green's functions. First of all, we easily derive the following lemma from standard elliptic theory:

Lemma 4.3. Let $\Omega$ be a smooth bounded domain in $\mathbb{R}^{3}$. Let $y \in \Omega$ and let $g$ be a weak solution in $H^{1}(\Omega)$ of

$$
\Delta g+h g=-\frac{h}{\omega_{2}|x-y|} \quad \text { in } \Omega .
$$

Then $g$ is continuous and can be written as

$$
g(x)=g(y)+\frac{h(y)}{2}|x-y|+\gamma_{y}(x) \quad \text { in } \Omega
$$

where $\gamma_{y} \in C^{1}(\Omega)$ satisfies $\gamma_{y}(y)=0$.

Applying the decomposition lemma to Green's functions, we get the following Pohožaev identity for their regular parts.

Lemma 4.4. Let $\Omega$ be a smooth bounded domain in $\mathbb{R}^{3}$, star-shaped with respect to 0 , and let $h \in C^{1}(\Omega)$ satisfy $[0.2)$. Let $\mathcal{G}_{h}$ be the Green function of $\Delta+h$. Let also $N \in \mathbb{N}^{*}$, $x_{1}, \ldots, x_{N} \in \Omega, \lambda_{1}, \ldots, \lambda_{N}$ some positive real numbers and

$$
G(x)=\sum_{i=1}^{N} \lambda_{i} \mathcal{G}_{h}\left(x, x_{i}\right)
$$

Then, using Lemma 4.3, we write $G$ in a neighbourhood of $x_{i}$ as

$$
G(x)=\frac{\lambda_{i}}{\omega_{2}\left|x-x_{i}\right|}+m_{i}+\lambda_{i} \frac{h\left(x_{i}\right)}{2}\left|x-x_{i}\right|+\gamma_{i}(x)
$$

where $m_{i} \in \mathbb{R}$ and $\gamma_{i} \in C^{1}(\Omega)$ satisfies $\gamma_{i}(0)=0$. Then

$$
\sum_{i=1}^{N} \lambda_{i}\left(m_{i}+2\left\langle x_{i}, \nabla \gamma_{x_{i}}\left(x_{i}\right)\right\rangle\right)<0
$$


Proof. We let $\delta>0$ be such that the $B\left(x_{i}, \delta\right)$ are disjoint and do not intersect the boundary of $\Omega$ and we set

$$
\Omega_{\delta}=\Omega \backslash \bigcup_{i=1}^{N} B\left(x_{i}, \delta\right) .
$$

Multiplying the equation satisfied by $G$ by $\langle x, \nabla G\rangle$, after some integrations by parts we obtain

$$
\begin{aligned}
\int_{\Omega_{\delta}}( & \left.\frac{1}{2}\langle x, \nabla h\rangle+h\right) G^{2} d x \\
= & \int_{\partial \Omega}\left(\frac{1}{2}\langle x, v\rangle\left(|\nabla G|^{2}+h G^{2}\right)-\left(\langle x, \nabla G\rangle+\frac{1}{2} G\right) \partial_{\nu} G\right) d \sigma \\
& \quad-\sum_{i=1}^{N} \int_{\partial B\left(x_{i}, \delta\right)}\left(\frac{1}{2}\langle x, v\rangle\left(|\nabla G|^{2}+h G^{2}\right)-\left(\langle x, \nabla G\rangle+\frac{1}{2} G\right) \partial_{\nu} G\right) d \sigma
\end{aligned}
$$

where $v$ denotes the outer normal to $\partial \Omega$ and to $\partial B\left(x_{i}, \delta\right)$ respectively. Noting that $G=0$ on $\partial \Omega$, we have

$$
\begin{aligned}
\int_{\partial \Omega}\left(\frac{1}{2}\langle x, v\rangle\left(|\nabla G|^{2}+h G^{2}\right)-\left(\langle x, \nabla G\rangle+\frac{1}{2} G\right) \partial_{\nu} G\right) d \sigma & \\
& =-\frac{1}{2} \int_{\partial \Omega}\langle x, v\rangle|\nabla G|^{2} d \sigma<0
\end{aligned}
$$

since $\Omega$ is star-shaped. Since $h$ satisfies 0.2 , we arrive at

$$
\sum_{i=1}^{N} \int_{\partial B\left(x_{i}, \delta\right)}\left(\frac{1}{2}\langle x, v\rangle\left(|\nabla G|^{2}+h G^{2}\right)-\left(\langle x, \nabla G\rangle+\frac{1}{2} G\right) \partial_{\nu} G\right) d \sigma \leq-C_{0}
$$

where $C_{0}$ is independent of $\delta$. It is easily checked that

$$
\int_{\partial B\left(x_{i}, \delta\right)}\langle x, \nu\rangle h G^{2} d \sigma \rightarrow 0 \quad \text { as } \delta \rightarrow 0 .
$$

In order to estimate the remaining terms, we write

$$
\begin{aligned}
\int_{\partial B\left(x_{i}, \delta\right)}\left(\frac{1}{2}\langle x, \nu\rangle|\nabla G|^{2}-\left(\langle x, \nabla G\rangle+\frac{1}{2} G\right) \partial_{\nu} G\right) d \sigma & \\
= & \int_{\partial B\left(x_{i}, \delta\right)}\left(\frac{1}{2}\left\langle x-x_{i}, v\right\rangle|\nabla G|^{2}-\left(\left\langle x-x_{i}, \nabla G\right\rangle+\frac{1}{2} G\right) \partial_{\nu} G\right) d \sigma \\
& +\int_{\partial B\left(x_{i}, \delta\right)}\left(\frac{1}{2}\left\langle x_{i}, v\right\rangle|\nabla G|^{2}-\left\langle x_{i}, \nabla G\right\rangle \partial_{\nu} G\right) d \sigma
\end{aligned}
$$

Then, thanks to the expansion of $G$ in a neighbourhood of $x_{i}$, one can easily check that

$$
\int_{\partial B\left(x_{i}, \delta\right)}\left(\frac{1}{2}\left\langle x-x_{i}, v\right\rangle|\nabla G|^{2}-\left(\left\langle x-x_{i}, \nabla G\right\rangle+\frac{1}{2} G\right) \partial_{\nu} G\right) d \sigma \rightarrow \frac{\lambda_{i}}{2} m_{i}
$$


and

$$
\int_{\partial B\left(x_{i}, \delta\right)}\left(\frac{1}{2}\left\langle x_{i}, v\right\rangle|\nabla G|^{2}-\left\langle x_{i}, \nabla G\right\rangle \partial_{\nu} G\right) d \sigma \rightarrow \lambda_{i}\left\langle x_{i}, \nabla \gamma_{x_{i}}\left(x_{i}\right)\right\rangle
$$

as $\delta \rightarrow 0$. Combining the above results gives the desired inequality.

Acknowledgments. The first author wishes to thank H. Brezis for drawing the question raised in [3] to his attention. This work was done while the two authors were staying at PIMS, Vancouver, for one year. They wish to thank all the members of PIMS for their great hospitality during that year.

\section{References}

[1] Bahri, A., Coron, J.-M.: On a nonlinear elliptic equation involving the critical Sobolev exponent: the effect of the topology of the domain. Comm. Pure Appl. Math. 41, 253-294 (1988) Zbl 0649.35033 MR 0929280

[2] Brezis, H., Nirenberg, L.: Positive solutions of nonlinear elliptic equations involving critical Sobolev exponents. Comm. Pure Appl. Math. 36, 437-477 (1983) Zbl 0541.35029 MR 0709644

[3] Brezis, H., Willem, M.: On some nonlinear equations with critical exponents. J. Funct. Anal. 255, 2286-2298 (2008) Zbl 1166.35021 MR 2473258

[4] Caffarelli, L. A., Gidas, B., Spruck, J.: Asymptotic symmetry and local behavior of semilinear elliptic equations with critical Sobolev growth. Comm. Pure Appl. Math. 42, 271-297 (1989) Zbl 0702.35085 MR 0982351

[5] Dancer, E. N.: Some notes on the method of moving planes. Bull. Austral. Math. Soc. 46, 425-434 (1992) Zbl 0777.35005 MR 1190345

[6] Druet, O.: Elliptic equations with critical Sobolev exponents in dimension 3. Ann. Inst. H. Poincaré Anal. Non Linéaire 19, 125-142 (2002) Zbl 1011.35060 MR 1902741

[7] Druet, O.: Compactness for Yamabe metrics in low dimensions. Int. Math. Res. Notices 2004, no. 23, 1143-1191 Zbl 1085.53029 MR 2041549

[8] Druet, O., Hebey, E.: Stability and instability for Einstein-scalar field Lichnerowicz equations on compact Riemannian manifolds. Math. Z. 263, 33-67 (2009) Zbl pre05598905 MR 2529487

[9] Gilbarg, D., Trudinger, N. S.: Elliptic Partial Differential Equations of Second Order. 2nd ed., Grundlehren Math. Wiss. 224, Springer, Berlin (1983) Zbl 0562.35001 MR 0737190

[10] Han, Q., Lin, F.: Elliptic Partial Differential Equations. Courant Lecture Notes in Math. 1, Courant Inst. Math. Sci., New York (1997) Zbl 1051.35031 MR 1669352

[11] Pohožaev, S. I.: On the eigenfunctions of the equation $\Delta u+\lambda f(u)=0$. Dokl. Akad. Nauk SSSR 165, 36-39 (1965) (in Russian) Zbl 0141.30202 MR 0192184 